\title{
Caractérisation et écologie des peuplements des mormyridae dans la réserve de Yoko (Kisangani, RD Congo)
}

\author{
Richard MAHAMBA BYANIKIRO ${ }^{1,2 *}$, Jacob NDJAKI NSILA ${ }^{2}$, \\ Alidor KANKONDA BUSANGA ${ }^{2}$, Joseph ULYELALI-PATHO ${ }^{2,3}$ et \\ Jean-Claude $\mathrm{MICHA}^{4}$ \\ ${ }^{I}$ Université de Goma, Faculté des Sciences Agronomiques, \\ Département de Phytotechnie, B.P : 204 Goma, R.D. Congo. \\ ${ }^{2}$ Université de Kisangani, Faculté des Sciences, \\ Département d'Hydrobiologie, B.P : 2012 Kisangani, R.D. Congo. \\ ${ }^{2,3}$ Université de Kisangani, Centre de Surveillance de la Biodiversité, \\ Département d'Ecologie et Biodiversité des Ressources Aquatiques, R.D. Congo. \\ ${ }^{4}$ Université de Namur (UN), Unité de Recherche en Biologie Environnementale (URBE), \\ rue de Bruxelles, 61 B-5000 Namur, Belgique. \\ * Auteur correspondant ; E-mail : mahambar@yahoo.fr ; Tél : (+243)810794509; (+243)853175676
}

\section{REMERCIEMENTS}

Nous pensons au projet REAFOR pour sa subvention, à l'Université de Namur/ Belgique.

\section{RESUME}

L'étude sur les peuplements de Morymyridae dans deux rivières forestières Yoko et Biaro à Kisangani, avait pour but de caractériser et d'étudier l'écologie des Mormyridae, l'une des familles bio-indicatrice qui pourra servir de monitoring futur en conservation et en aménagement. Elle a été conduite de septembre 2008 à août 2009 en utilisant des filets maillants, des palangres et des nasses traditionnelles pour les récoltes des poissons. La caractérisation des habitats a été faite selon une fiche de suivi hydromorphologique en précisant les profils transversaux des rivières tous les $50 \mathrm{~m}$ pour chacune des 8 stations sélectionnées. 214 spécimens de poissons appartenant à 7 espèces et 4 genres ont été récoltés. L'espèce Stomatorhinus corneti est la plus représentée avec 144 spécimens soit $67,3 \%$, suivie de Petrocephalus microphtalmus avec 50 individus soit 23,4\%. Les richesses taxinomiques les plus élevées ont été observées dans les stations Yoko 4 (6 espèces) et Biaro 3 (5 espèces) et des indices de diversité et d'équitabilité élevés. Les AFC montrent qu'il existe des liens nets entre les variables environnementales : largeur, profondeur, oxygène dissous, vitesse du courant d'eau, végétation riveraine, nature du substrat (sablo-argileux, caillouteux, graveleux, vaseux, etc.) et que les espèces S. corneti et P. microphtalmus sont fortement liées aux stations Yoko 2 et 3, Biaro 1, 2 et 4 en corrélation positive entre ces variables et les axes I et II.Ceci montre, indubitablement, que les communautés de poissons sont réparties sur la base d'un ensemble complexe d'interrelations entre les différentes variables abiotiques et biotiques qui décrivent l'environnement, entre autres, le substrat (minéral ou végétal), la profondeur, l'oxygène dissous, etc.

(C) 2017 International Formulae Group. All rights reserved.

Mots clés : Habitats, diversité, Mormyridae, distribution spatiale, rivières Yoko et Biaro. 


\title{
Characterization and ecology of stands of Mormyrids in the Reserve of Yoko (Kisangani, DR. Congo)
}

\begin{abstract}
Study on the Mormyridae stands in two forest rivers Yoko and Biaro near Kisangani, aimed at characterizing and studying the ecology of the Mormyridae one family of bio-indicator that can be used in conservation and monitoring. It was conducted from September 2008 to August 2009, using gillnets, longlines and traditional traps for the harvest of fish. The characterization of Habitat was made according to a morphology follow - up from specifying transverse profiles every $50 \mathrm{~m}$ for each of the 8 selected stations. 214 specimens of fish belonging to 7 species and 4 genera were harvested. Stomatorhinus corneti species is the most represented with 144 specimens either $67.3 \%$, followed by Petrocephalus microphtalmus with 50 individuals or $23.4 \%$. The highest taxonomicrichness was observed in stations Yoko 4 (6 species) and Biaro 3 (5 species) and high diversity and equitability indices. The AFC show that there are net links between environmental variables: width, depth, dissolved oxygen, current speed of water, vegetation, nature of the substrate (Sandy - clayey, stony, gravelly, etc.) and that S. corneti and P. microphtalamus species are strongly linked to the stations Yoko 2 and 3, Biaro 1, 2 and 4 in positive correlation between these variables and axes I and II. This undoubtedly shows that fish communities are distributed based on a complex set of interrelationships between different abioc and biotic variables that describe the environment among others the substrate (mineral or vegetable), depth, oxygen dissolved, etc.
\end{abstract}

(C) 2017 International Formulae Group. All rights reserved.

Keywords: Habitats, diversity, Mormyridae, spatial distribution, Yoko and Biaro rivers.

\section{INTRODUCTION}

La notion de « biodiversité » est fortement médiatisée actuellement et l'on s'inquiète de la disparition de différentes espèces connues ou non. Malgré cela, certains groupes d'organismes comme les poissons d'eau douce d'Afrique centrale, restent mal connus, or, ils présentent une diversité remarquable (Sullivan et al., 2004). C'est pourquoi diverses études essayent de définir et de préciser cette diversité ichtyologique, entre autres, pour pouvoir la protéger le cas échéant (Sullivan et Hopkins, 2001). En effet, il se pose la question « comment sauvegarder cette multitude d'espèces si on ne la connaît pas »?

La famille des Mormyridae, endémique à l'Afrique subsaharienne, constitue une très grande famille panafricaine comptant actuellement vingt et un genres et 221 espèces, dans le monde. Certaines peuvent mesurer jusqu'à $1,5 \mathrm{~m}$ de long quand bien même la majorité a une taille comprise entre 9 et $50 \mathrm{~cm}$. Ces poissons sont souvent grégaires, formant des bancs dans les eaux troubles des rivières. Ce comportement et adaptation est probablement favorisé par la physiologie très particulière de leurs organes sensoriels (Kramer, 2009).

La région de Kisangani regorge de cours d'eau forestiers tropicaux appartenant au bassin du Congo et regroupant une très grande diversité ichtyologique qui, elle-même n'est pas encore bien connue sur le plan taxonomique et encore moins sur le plan de la biologie et de l'écologie des espèces qui les peuplent. Ces lacunes ne peuvent être comblées que par des études locales et régionales des différents groupes de poissons qui colonisent notamment les petits cours d'eau forestiers rarement prospectés jusqu'à présent. Nous nous sommes donc intéressés à deux petits cours d'eau forestiers, Yoko et Biaro, pour y effectuer la caractérisation des peuplements des Mormyridae qui sont considérées par plusieurs auteurs comme étant parmi les premiers poissons à réagir lors d'altérations du milieu et, par conséquent, pourraient être considérés comme de bons bio 
- indicateurs pour caractériser l'état présent d'un habitat-clé (Dale et Beyeler, 2001; Niemi et McDonald, 2004), ainsi que l'évolution de l'état de l'écosystème dans le temps (Lévêque et Mounolou, 2008 ; Niamien-Ebrottié et al., 2008).

Ainsi, les Mormyridae et leurs espèces indicatrices potentielles pourraient servir de monitoring dans l'évaluation de la biodiversité de la communauté actuelle et future (Kerckhove, 2012). Toute mesure de conservation et d'aménagement à prendre devient, dès lors, une préoccupation majeure et devrait reposer sur les données scientifiques les plus fiables disponibles et être conçue pour assurer l'utilisation durable des ressources halieutiques. Ces mesures, pour être efficaces, nécessitent une bonne connaissance des espèces et des relations qui les lient à leur milieu (Lalèyè et al., 2004). Les études de la biologie et de l'écologie des espèces doit donc occuper une place de choix dans la conservation et la surveillance des peuplements icthyologiques (Mbimbi, 2006).

Nous pensons que les rivières de la forêt tropicale inondable congolaise sont fortement colonisées par des espèces particulières de Mormyridae qui s'y sont diversifiées en s'adaptant aux habitats particuliers existants. L'étude de la structure spatio-temporelle des peuplements des Mormyridae, basée sur la diversité des habitats, devrait nous éclairer à cet égard.

\section{MATERIEL ET METHODES Milieu d'étude}

La présente étude a été menée dans les rivières Yoko et Biaro en Territoire d'Ubundu, District de la Tshopo, Province Orientale (Figure 1), RD Congo. La rivière Yoko a une longueur totale $13 \mathrm{~km}$ à vol d'oiseau. Elle est traversée par la route menant vers Ubundu à $27 \mathrm{~km}$ de la ville de Kisangani. Ses coordonnées géographiques sont les suivantes: latitude Nord : $00^{\circ} 17^{\prime} 17,0^{\prime \prime}$; longitude Est : $025^{\circ} 16^{\prime} 24,3^{\prime \prime}$, l'altitude est de $403 \mathrm{~m}$, son bassin versant est directement situé dans la Réserve de Yoko (Figure 1). Cette réserve a une superficie globale de 6.975 ha. Quant à la rivière Biaro, elle prend sa source dans la forêt de Batiabetu à $58 \mathrm{~km}$ de la ville de Kisangani. Elle a une longueur totale de 40,2 $\mathrm{km}$ à vol d'oiseau. Elle se jette dans le Fleuve Congo à Babokoti dans la collectivité secteur de Lubuya- Bera. La rivière est traversée par la route Kisangani - Ubundu à $38 \mathrm{~km}$ de Kisangani. Elle limite la Réserve de Yoko dans sa partie Sud - Est et forme une demi - boucle en suivant cette direction (Kumba et al., 2013). Ses coordonnées géographiques sont les suivantes: latitude Nord $00^{\circ} 15,17,2$ "'; longitude Est $025^{\circ} 18^{\prime}$ '52,5' et l'altitude est de $402 \mathrm{~m}$ (Figure 1). La Figure 1 montre les différentes stations et la description de l'occupation du sol de cette recherche.

\section{Echantillonnage et analyse}

Huit stations ont été choisies dans les rivières Yoko et Biaro, dont quatre sont localisées dans la Réserve de Yoko et les quatre autres en dehors de la Réserve. Les échantillons de Mormyridae ont été récoltés de septembre 2008 à août 2009 à l'aide des différentes techniques: filets maillants de 20 $\mathrm{m}$ de long et de $1 \mathrm{~m}$ de hauteur et dont les mailles entrenœuds sont de $1,5 \mathrm{~cm}, 2 \mathrm{~cm}, 2,5$ $\mathrm{cm}$ et $3 \mathrm{~cm}$; des nasses traditionnelles d'environ $1 \mathrm{~m}$ de long et $30 \mathrm{~cm}$ de diamètre, et des lignes individuelles et des palangres de $25 \mathrm{~m}$ de long avec hameçons numéros: 8 ; $10 ; 12 ; 14 ; 16 ; 18$ et 20 ont été appâtés de vers de terre, de mollusques, de petits poissons, etc. Les pêches ont toutes été effectuées dans la troisième semaine de chaque mois. Sur le terrain, les engins et la technique de pêche étaient standardisés pour toutes les stations et les travaux de terrain débutaient au même moment dans l'ensemble des stations. L'échantillonnage des poissons était réalisé dans tous les cas sur base d'une fréquence cumulée de trois nuits d'effort de capture par station et par mois durant une période de douze mois. La technique consistait à mettre en place les filets et lignes de pêche face au courant d'eau dans les différentes stations. Ces derniers étaient placés entre 17 et $18 \mathrm{~h} 00$ ' le soir et les relevés entre 6 et $7 \mathrm{~h} 00^{\prime}$ du matin. 
Après le relevé des filets, les spécimens récoltés étaient conservés dans des bocaux en plastique de quatre litres contenant une solution de formol à 4\%. Les échantillons ainsi constitués étaient étiquetés selon les mois de récolte et par station, puis amenés au Laboratoire d'Hydrobiologie de la Faculté des Sciences de l'Université de Kisangani pour une identification ultérieure.

$\mathrm{Au}$ Laboratoire, les échantillons conservés au formol ont été déformolisés, regroupés et identifiés en suivant les périodes de récolte par station et par mois grâce aux clés de détermination de : Poll et Gosse (1995), Paugy et al. (2003), Mbega (2004), Abell et al. (2008).

\section{Caractérisation du milieu}

Les critères utilisés en délimitant les profils transversaux tous les $50 \mathrm{~m}$ de long à chaque station (caractérisation des habitats) sont ceux préconisés dans la fiche de monitoring hydromorphologique proposée par Verniers et al. (2009) et l'Agence de l'eau Rhin Meuse (2000). Ils consistaient en : largeur du lit du cours d'eau mesurée à l'aide d'un penta décamètre; profondeur de l'eau prise in situ à l'aide d'un appareil de mesure de profondeur de marque Kritech (précision 1 $\mathrm{cm}$ ) ; vitesse du courant d'eau mesurée grâce à un Courantomètre (type 281D) (précision $0,1 \mathrm{~cm} / \mathrm{sec}$ ) ; nature de fond (sable, graviers, vases, bois morts, végétation herbacée,...), forme du lit; nature des berges par une observation directe à l'œil nu; espèces végétales identifiées directement sur le terrain selon le Catalogue - flore des plantes vasculaires des districts de Kisangani et de la Tshopo (RD. Congo) (Lejoly et al., 2010). Toutefois, un herbier a été constitué avec les spécimens douteux, amenés alors à l'herbarium de la Faculté des Sciences de l'Université de Kisangani, pour l'identification précise. Les coordonnées géographiques de chaque station étaient prélevées à l'aide d'un appareil GPS de marque Garmin 60cx ; la conductivité, le taux de salinité, la température et le $\mathrm{pH}$ de l'eau étaient mesurés sur le terrain à l'aide d'un Combo de marque Hanna (précision respective : $\mathrm{K}_{20}: 2 \mu \mathrm{S} / \mathrm{cm}, 0,1 \mathrm{ppm}, 0,1{ }^{\circ} \mathrm{C}$, $0,1)$; L'oxygène dissous (concentration et saturation) était mesuré in situ à l'aide d'un oxymètre de marque Oxygard (précision 0,1 $\left.\mathrm{mg} \mathrm{l}^{-1}\right)$.

\section{Analyses statistiques et traitement des données}

- Les Analyses en Composantes Principales (ACP) ont été appliquées en vue de voir si les données recueillies présentaient une quelconque ordination du milieu. Les données correspondaient à une matrice brute des mesures effectuées dans les stations de recherche pour 9 variables physico-chimiques enregistrées une fois par station et par mois.

- L'Analyse Factorielle des Correspondances (AFC) (Jongman et al., 1995) a été utilisée pour traiter les données relatives à la distribution spatio-temporelle des peuplements ichtyologiques des Mormyridae dans les rivières Yoko et Biaro.

- L'analyse de la variance à un critère de classification (ANOVA 1) a été utilisée afin de tester le degré de signification d'éventuelles différences dans les comparaisons effectuées entre les structures spatio-temporelles mises en évidence par les techniques d'analyses multivariées (AFC).

Les indices de diversité suivants ont également été utilisés pour caractériser les peuplements ichtyologiques des Mormyridae :

Indice de diversité de Shannon s'exprime par la relation suivante : $H^{\prime}=-\Sigma$ $(n i / N) \ln (n i / N)$, où $H^{\prime}=$ indice de Shannon \& Weaver (1949) ; ni = nombre d'individus du taxon $\mathrm{i} ; \mathrm{N}=$ nombre total d'individus de la population.

L'équitabilité, rapport de la diversité réelle à la diversité maximale, s'obtient en divisant l'indice de diversité de Shannon par le logarithme en base 2 de la richesse spécifique (Piélou, 1969). Selon Dajoz, (1996), la formule utilisée est la suivante : E $=\mathrm{H}^{\prime} / \log 2 \mathrm{~S}$. Où $\mathrm{E}=$ équitabilité; $\mathrm{H}^{\prime}=$ indice de Shannon \& Weaver (1949); S = richesse spécifique.

- Indice de diversité (1-D) de Simpson est représenté par 1-D, le maximum de diversité étant représenté par la valeur 1 , et 
le minimum de diversité par la valeur 0 (Bütler, 2000). $D=\Sigma f i^{2}$ Où : fi $=\mathrm{ni} / \mathrm{N}$; ni : nombre d'individus de l'espèce donnée; $\mathrm{N}$ : nombre total d'individus.

L'indice de similarité de Jaccard (Legendre et Legendre, 1998 ; Angelier, 2000): $I S=N c^{*} 100 / N a+N b-N C$ est utilisé en vue de comparer les différents types d'échantillonnage sur la base de la présence/absence des taxons. IS $=$ indice de Jaccard ; $\mathrm{Nc}=$ nombre de taxons communs aux deux stations; $\mathrm{Na}$ et $\mathrm{Nb}=$ nombre total de taxons dans nos stations.

Pour ce faire, les programmes suivants nous ont servis pour effectuer l'ensemble des calculs : le logiciel R 2.5.0; Biodiversity \& MVSP (BiodivR1.0) et Past; le logiciel Excel 2003 et 2007.

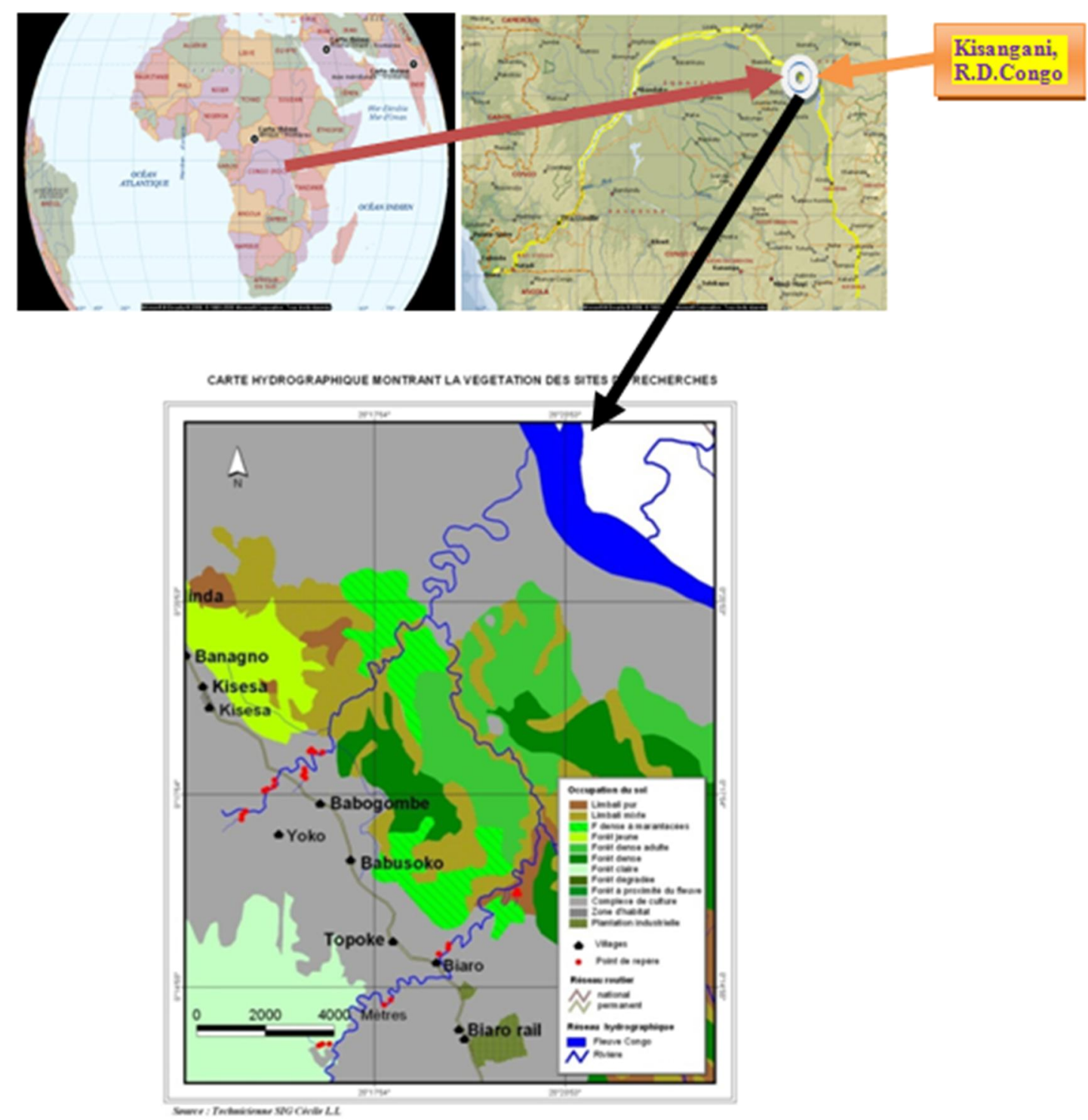

Figure 1 : Carte hydrographique avec 8 stations (points rouges) dans les rivières Yoko et Biaro (numérotées 1, 2, 3, 4 de l'amont vers l'aval) affluentes du fleuve Congo. Caractérisation hydromorphologique, végétale et ichtyologique (Mormyridae). 


\section{RESULTATS}

\section{Examen des biotopes des 8 stations de prélèvements}

D'une manière générale, les résultats $\mathrm{du}$ Tableau 1 montrent que la largeur moyenne annuelle sous eau des stations prospectées, pour toute la période d'étude, varie de 6,69 à $18,32 \mathrm{~m}$. Les grandes valeurs moyennes de la largeur s'observent aux stations Biaro $3(18,32 \mathrm{~m})$ et Biaro $2(17,85$ $\mathrm{m})$, et aux stations Yoko $4(15,6 \mathrm{~m})$ et Yoko 2 $(14,77 \mathrm{~m})$ situées dans les principaux cours d'eau respectivement Biaro et Yoko, tandis que les petites valeurs moyennes annuelles s'observent aux stations localisées dans les affluents notamment la station Biaro $1(6,69$ m) dans la rivière Amafombo et Biaro $4(8,00$ $\mathrm{m})$ dans la rivière Kenge ainsi qu'à la station Yoko 1 dans la rivière Losongo $(8,14 \mathrm{~m})$.

Dans l'ensemble des stations, la valeur moyenne annuelle de profondeur de l'eau pour toute la période annuelle de cette étude varie de 20,17 à $70,30 \mathrm{~cm}$. Elle est élevée aux stations Biaro $3(70,30 \mathrm{~cm})$ et Biaro $2(66,90$ $\mathrm{cm})$, et aux stations Yoko $4(47,95 \mathrm{~cm})$ et Yoko $2(38,16 \mathrm{~cm})$ situées dans les principaux cours d'eau (Biaro et Yoko), tandis que les valeurs faibles s'observent aux stations localisées dans les affluents notamment la station Yoko $1(20,17 \mathrm{~cm})$ dans la rivière Losongo et Biaro $1(28,47 \mathrm{~cm})$ dans la rivière Amafombo.

La teneur moyenne annuelle en oxygène dissous de l'eau durant cette étude semble varier moins d'une station à l'autre. Elle varie de 4,80 à $5,56 \mathrm{mg} / \mathrm{l}$. Elle a tendance à diminuer à la station Yoko $1(4,80 \mathrm{mg} / \mathrm{l})$.

Les moyennes générales annuelles de la température pour l'ensemble des stations n'ont pas beaucoup varié au cours de l'année pour cette étude. Elles oscillent autour de 24 ${ }^{\circ} \mathrm{C}$ en général. Elles sont légèrement plus faibles aux stations Biaro 4 avec $23,15{ }^{\circ} \mathrm{C}$, Yoko 3 avec 23,34 ${ }^{\circ} \mathrm{C}$ et Biaro 1 avec 23,42 ${ }^{\circ} \mathrm{C}$ localisées respectivement dans les affluents Kenge en forêt primaire, Mungamba et Biaro en forêt secondaire adulte.

$\mathrm{La}$ valeur moyenne annuelle de saturation de l'eau a varié de 73,87 à $86,89 \%$ pour l'ensemble des stations. Elle a tendance à augmenter au fur et à mesure que l'eau coule vers l'aval, soit $86,89 \%$ à la station Yoko 4 ; $86,39 \%$ à la station Yoko 3 et $84,47 \%$ à la station Biaro 4 ; elle est légèrement plus faible en amont soit $73,87 \%$ à la station Yoko 1 ; $76,79 \%$ à la station Biaro 1 et $78,14 \%$ pour la station Biaro 3.

Les valeurs moyennes générales annuelles de la salinité (ppm) de l'eau pour l'ensemble des stations montrent que la salinité moyenne de l'eau pour toute la période de cette étude a varié de 38,58 à 61,06 ppm. La valeur maximale s'observe à la station Yoko 1 dans la rivière Losongo avec $61,06 \mathrm{ppm}$ en moyenne de salinité et la valeur minimale à la station Biaro 2 avec 38,58 ppm en moyenne dans la rivière Biaro.

Les conductivités moyennes (standardisées à $20^{\circ} \mathrm{C}$ ) obtenues durant une année montrent que, dans l'ensemble des stations, les valeurs moyennes annuelles ont varié de 82,95 à $121,04 \mu \mathrm{S} / \mathrm{cm}$. Elles sont plus élevées aux stations Yoko 1 avec 121,04 $\mu \mathrm{S} / \mathrm{cm}$ et Yoko 2 avec 108,67 $\mu \mathrm{S} / \mathrm{cm}$; tandis que les valeurs les plus faibles sont observées aux stations Biaro 3 et 2 avec respectivement $82,95 \mu \mathrm{S} / \mathrm{cm}$ et $86,61 \mu \mathrm{S} / \mathrm{cm}$.

Les valeurs moyennes annuelles de $\mathrm{pH}$ de l'eau durant la période de cette étude sont généralement basiques pour l'ensemble des stations et elles oscillent autour de 8. Les valeurs maximales sont observées aux stations Yoko $2(8,13)$ et Biaro 3 avec 8,02 ; tandis que les valeurs minimales aux stations Yoko 1 et Biaro 2 avec chacune 7,87.

La vitesse moyenne annuelle de l'eau par station n'a pas beaucoup varié $(0,29$ à 0,57 $\mathrm{m} / \mathrm{s}$ ) durant la période annuelle de cette étude. Elle a tendance à être plus élevée à la station Yoko $4(0,57 \mathrm{~m} / \mathrm{s})$ et plus faible à la station Biaro $4(0,29 \mathrm{~m} / \mathrm{s})$. Dans l'ensemble, l'eau est claire pour toutes les stations mais les substrats benthiques varient d'une station à une autre.

Les résultats globaux du Tableau 2 montrent au total que 195 espèces végétales regroupées en 56 familles ont été inventoriées dans l'ensemble des 8 stations (berges, rives et fond) des rivières Biaro et Yoko. Les Fabaceae (28 espèces), les Malvaceae (14 
espèces), les Euphorbiaceae (11 espèces) et les Marantaceae (10 espèces) dominent en diversité et sont suivies des Poaceae (8 espèces), des Meliaceae ( 7 espèces) et des Annonaceae, Commelinaceae, Moraceae, Rutaceae, Connaraceae avec chacune 5 espèces puis des familles des Arecaceae, Sapotaceae, Salicaceae, Cyperaceae avec chacune 4 espèces. Par ailleurs, les résultats montrent que, certaines familles et espèces sont réparties dans toutes les stations et que, d'autres sont limitées à l'une ou l'autre station (Figure 2).

Pour ce qui concerne la rivière Biaro, les résultats indiquent que, sur le total des 56 familles inventoriées dans l'ensemble des stations, 27 familles et 54 espèces ont été observées à la station Biaro 1. Les Fabaceae (6 espèces) et les Urticaceae ( 3 espèces) sont les mieux représentées dans la strate supérieure suivies des Apocynacaeae, des Arecaceae et des Moraceae avec chacune 2 espèces. Par contre, la strate moyenne est dominée par les Euphorbiaceae (3 espèces) et la strate inférieure ainsi que la ripisylve sont dominées par les Marantaceae et les Poaceae avec chacune 4 espèces suivies des Commelinaceae (3 espèces). Les espèces Pseudospondias microcarpa, Cynometra alexandri, Macaranga saccifera et les Marantaceae sont les plus abondantes dans cette station (Figure 2).

La station Biaro 2 présente la même allure avec 30 familles et 78 espèces du total général. Les Fabaceae (6 espèces) et les Arecaceae (3 espèces) dominent la strate supérieure. Les Euphorbiaceae (3 espèces) représentent la strate intermédiaire. Par contre, la strate inférieure et la ripisylve sont dominées par les Marantaceae (6 espèces) suivie des Commelinaceae et des Poaceae avec chacune 4 espèces ainsi que des Solanaceae (3 espèces). Les espèces $P$. microcapa et Sacrophrinium megalophrinium sont les plus abondantes dans cette station.

Par ailleurs, les résultats montrent qu'à la station Biaro 3, 35 familles et 74 espèces du total général ont été inventoriées dans cette station, et que ce sont les Fabaceae (7 espèces), suivie des Euphorbiaceae (4 espèces) et des Urticaceae (3 espèces) qui sont les plus représentées à la strate supérieure. A la strate intermédiaire, ce sont les Fabaceae (5 espèces) qui dominent. Par contre, à la strate inférieure ainsi qu'en ripisylve, ce sont les Marantaceae (4 espèces), les Poaceae et les Thelypteridaceae avec chacune 2 espèces qui y sont les mieux représentées. Les espèces $P$. microcarpa, S. megalophrinium, longent les rives de la station de manière abondante (Figure 2).

Contrairement aux stations précédentes, il se fait remarquer qu'à la station Biaro 4, 33 familles et 68 espèces du total général sont présentes, et que les Fabaceae et les Malvaceae avec chacune 4 espèces, suivie des Rubiaceae avec 3 espèces sont les plus diversifiées aux strates supérieure et moyenne. Par ailleurs, à la strate inférieure et en ripisylve, ce sont les familles des Commelinaceae (6 espèces) et des Marantaceae (4 espèces) qui dominent. Les espèces $G$. dewevei, P. microcarpa, J. seretii et $C$. alexadri ainsi que les Marantaceae sont abondantes dans cette station (Figure 2).

Pour la rivière Yoko, les résultats renseignent qu'à la station Yoko 1 que, 36 familles et 61 espèces du total général ont été répertoriées. Les Fabaceae (8 espèces) suivie des Malvaceae et des Meliaceae avec chacune 3 espèces sont les plus représentées à la strate supérieure tandis qu'à la strate intermédiaire, ce sont les Fabaceae avec 4 espèces qui dominent. Par contre, à la strate inférieure, ainsi qu'en ripisylve, ce sont les Marantaceae (6 espèces) et les Commelinaceae (4 espèces) qui sont les plus diversifiées.

En ce qui concerne la station Yoko 2, 30 familles et 52 espèces ont été observées sur un total général des 56 familles inventoriées dans l'ensemble des stations, dont les Fabaceae (4 espèces), les Urticaceae et les Meliaceae avec chacune 2 espèces dominent la strate supérieure. La strate intermédiaire est représentée par les Arecaceae avec 3 espèces les Euphorbiaceae, les Fabaceae et les Rubiaceae avec chacune 2 espèces. En strate inférieure et en ripisylve, dominent les Commelinaceae, Cyperaceae, Marantaceae et Poaceae avec chacune 3 espèces. Les espèces 
P. microcapa et G. kisantuense sont les plus abondantes et longent harmonieusement cette station (Figure 2).

Quant en ce qui concerne la station Yoko 3, 35 familles et 63 espèces du total général des familles inventoriées dans l'ensemble de stations sont présentes, les Euphorbiaceae, les Fabaceae, les Meliaceae et les Malvaceae avec chacune 3 espèces sont les plus représentées à la strate supérieure. Par contre, à la strate moyenne, ce sont les familles des Fabaceae (6 espèces) suivie des Salicaceae ( 2 espèces) qui y sont les plus diversifiées. A la strate inférieure, ainsi qu'à la ripisylve, ce sont les Commelinaceae, les Marantaceae et les Poaceae avec chacune 3 espèces qui y sont les plus représentées. Les espèces $P$. microcarpa et Bambusa vilgaris sont caractéristiques de la station (Figure 2).

Enfin, à la station Yoko 4, 28 familles et 59 espèces du total général des familles inventoriées dans l'ensemble des stations sont présentes. Les Fabaceae (6 espèces), suivies des Meliaceae et des Malvaceae avec chacune 3 espèces sont les plus diversifiées à la strate supérieure. A la strate moyenne, ce sont les Fabaceae (6 espèces) suivies des Euphorbiaceae (4 espèces) et des Meliaceae (3 espèces) qui sont les plus diversifiées. Par contre, à la strate inférieure ainsi qu'en ripisylve, ce sont les Commelinaceae avec 2 espèces qui sont les plus représentées. Les espèces $P$. microcarpa, $G$. dewevrei longent les rives de cette station de manière importante (Figure 2).

\section{Caractérisation et composition faunistique des peuplements de Mormyridae}

$\mathrm{Au}$ cours d'une année complète, huit stations des rivières Biaro et Yoko ont été prospectées mensuellement (Tableau 3). Deux cent quatorze spécimens de poissons Mormyridae au total ont été récoltés, répartis en 7 espèces appartenant à 4 genres. L'espèce Stomatorhinus corneti est la plus abondante avec 144 spécimens représentant $67,3 \%$, suivie de Petrocephalus microphtalmus avec 50 individus, soit 23,4\%. Les stations Biaro 2 et Biaro 1 (Tableau 4) contiennent les plus grands nombres d'individus représentant respectivement $30,8 \%$ et $28,0 \%$ alors que les stations Yoko 2 et Yoko 3 ne présentent aucun individu.

Le Tableau 5 révèle que la richesse taxonomique la plus élevée est observée aux stations Yoko 4 et Biaro 3 avec respectivement 6 et 5 espèces, ce qui se traduit par des indices de diversité et d'équitabilité élevés. Les stations Yoko 2 et 3 ne présentent aucun individu.

\section{Distribution spatiale des espèces de Mormyridae dans les 8 stations \\ Les résultats des AFC (Figure 4) sur la} répartition spatiale des espèces capturées par station ainsi que leurs effectifs (Tableau 3 ) ont permis de sélectionner les variables environnementales et biotiques qui expriment au mieux les liens qui existent entre espèces variables et les stations. Dans les stations Yoko 2 et 3, Biaro 1, 2 et 4, les variables environnementales qui influencent la distribution des espèces de Mormyridae sont : la végétation, la température de l'eau, la vitesse de l'eau, la profondeur de l'eau, l'oxygène dissous dans l'eau et la nature des substrats qui sont positivement corrélées aux axes I et II ; à ces variables s'associent les espèces de Mormyridae, $S$. corneti, $P$. microphtalmus, qui ont plus de chances d'être capturées dans l'ensemble de ces stations. L'espèce $P$. pallidomaculatus a plus d'affinité avec la station Biaro 3 due à la corrélation positive qui existe entre l'axe I et la largeur, la profondeur, la saturation, la végétation ripicole ainsi que le substrat qui est sabloargileux, rocheux avec bois morts et débris végétaux. L'unique espèce Marcusenius cyprinoides capturée à Yoko 1 (petit affluent en dehors de la réserve) est influencée en axe II par la profondeur, l'oxygène dissous associés à la végétation et à la nature du substrat qui est sablonneux, rocheux, caillouteux et encombré de branchages.

Enfin, l'espèce Marcusenius senegalensis, à la station Yoko 4, est influencée par la végétation, la vitesse de l'eau, la profondeur, la saturation en oxygène ainsi que la nature du substrat qui est sablonneux, limoneux, graveleux (axe II). 
L'indice de similarité euclidienne et la distance de Bray-Curtys (Tableau 6 et Figure 5) montrent une démarcation de deux grands pools écologiques (Yoko et Biaro) dont un se subdivise en trois axes principaux ayant des caractéristiques différentes aux seuils d'environ 44 et 17 de l'échelle. De fortes similitudes s'observent entre Biaro 1 et 2 au seuil 8 environ, et entre Yoko 1, 2 et 3 au seuil d'environ 2 car ces stations se trouvent en dehors de la réserve et sont en forêts secondaires subissant des perturbations dues aux activités humaines. Yoko 4 et Biaro 3 et 4 sont similaires au point de l'échelle avoisinant 9 car, contrairement aux autres stations, ces dernières se trouvent en pleine réserve et dans une vieille forêt secondaire où les conditions écologiques sont stables.

Les résultats des ACP (Figure 3) relatifs aux facteurs hydromorphologiques montrent que la conductivité est fortement corrélée à la station Yoko 1, la température à Yoko 2, la vitesse à Yoko 4, la largeur à Biaro 3 , la profondeur à Biaro 2, la saturation en oxygène à Yoko 3 et à Biaro 4, paramètres qui influencent la répartition des poissons dans ces stations. On distingue ainsi 4 entités différentes. La première comprend les stations Yoko 1 et Biaro 1 qui se ressemblent (forme $\mathrm{du}$ lit majeur et largeur presque semblables, vitesse du courant d'eau de $0,35 \mathrm{~m} / \mathrm{sec}$ en moyenne), situées en dehors de la réserve traversant des jachères, des recrus forestiers, des champs et des forêts secondaires adultes où dominent les Annacardiaceae, Annonaceae, Apocynaceae, Areacaeae, Fabaceae et Meliaceae en strate supérieure ; les Costaceae, Euphorbiaceae, Fabaceae, Commelinaceae et Connaraceae en strate intermédiaire et les Cyperaceae, Loganiaceae, Marantaceae et Poaceae en ripisylve (Tableau 2). Ceci contribue à la modification de l'habitat et des microhabitats des rivières, notamment à la réduction de l'oxygène dissous $(<5 \mathrm{mg} / \mathrm{l})$. Il en résulte qu'une seule espèce de Mormyridae qui est présente dans chacune de ces stations: Marusenius cyprinoides en Yoko 1 et Stomatorhinus corneti en Biaro 1.
La seconde entité (Figure 3) comprend Biaro 2 et 3 et Yoko 4 qui se ressemblent fort, à tel point que Yoko 4 et Biaro 3 sont similaires car situées en aval des principales rivières et en pleine réserve de la Yoko, elles renferment les peuplements ichtyologiques les plus diversifiés, dus à la diversification des microhabitats. Les valeurs des paramètres profondeur, oxygène dissous, nature $\mathrm{du}$ substrat (Tableau 1), démontrent que les caractéristiques de ces stations sont fort semblables et expriment bien les corrélations entre espèces de poisson et variables environnementales. Ainsi Biaro 3 et Yoko 4 (Tableau 2, Figures 1 et 2) situées en pleine réserve dans une vieille forêt secondaire présentent les mêmes familles végétales: Anacardiaceae, Annonaceae, Apocynaceae, Ebenaceae, Euphorbiaceae, Fabaceae, Meliaceae, Moraceae, Mysticaceae, Strombosiaceae, Sapindaceae et Malvaceae en strate supérieure; Arecaceae, Dichapetalaceae, Dilleniaceae, Euphorbiacea, Fabaceae Salicaceae, Vitaceae, Passifloraceae, Zingiberaceae, Araceae, Combretaceae et Commelinaceae en strate intermédiaire et Marantaceae, Poaceae, Smilacaceae, Thelypteridaceae en ripisylve.

$\mathrm{La}$ troisième entité (Figure 3) est constituée de Biaro 4 et Yoko 3 situées dans deux différents affluents où des ressemblances sont observées entre les valeurs des paramètres oxygène dissous dans l'eau, saturation de l'eau, nature du substrat etc. (Tableau 1), démontrent que ces stations sont fort semblables. Ce deux affluents situés en dehors de la réserve traversant des forêts secondaires adultes, des recrus forestiers, mais surtout des champs et des jachères où dominent les Fabaceae, Malvaceae, Meliaceae, Rubiaceae et Salicaceae en strates supérieure et moyenne, et qu'en ripisylve ce sont les Commelinaceae, Marantaceae et les Poaceae qui y dominent (Tableau 2). Ceci contribue à la modification de l'habitat et des microhabitats des rivières. Les deux espèces de Mormyridae capturées à Biaro 4 Petrocephalus microphtalmus et Stomatorhinus corneti émane de la période ainsi que de l'exploration hasardeuse de ces 
poissons dans la zone de confluence située entre la forêt secondaire adulte de la réserve et les jachères (Tableau 4 et Figures 1et 2).

Enfin, l'entité Yoko 2 ne présente aucune similarité avec d'autres stations et aucune espèce de Mormyridae n'y a été signalée. Car, cette station se situe en dehors de la réserve, à proximité de la route Kisangani-Ubundu et est donc soumise à de fortes activités anthropiques (champs, bois de chauffe, piègage, écopage, etc.), résultant en jachères et recrus forestiers, où l'on observe une abondance des Commelinaceae, Cyperaceae, Marantaceae et Poaceae en ripisylve, occasionnant la modification de paramètres physico-chiques; tels que la conductivité $(108,67 \mu \mathrm{S} / \mathrm{cm})$ et la température $\left(24,03{ }^{\circ} \mathrm{C}\right)$ etc. (Tableau 1 et Figures 1et 2).
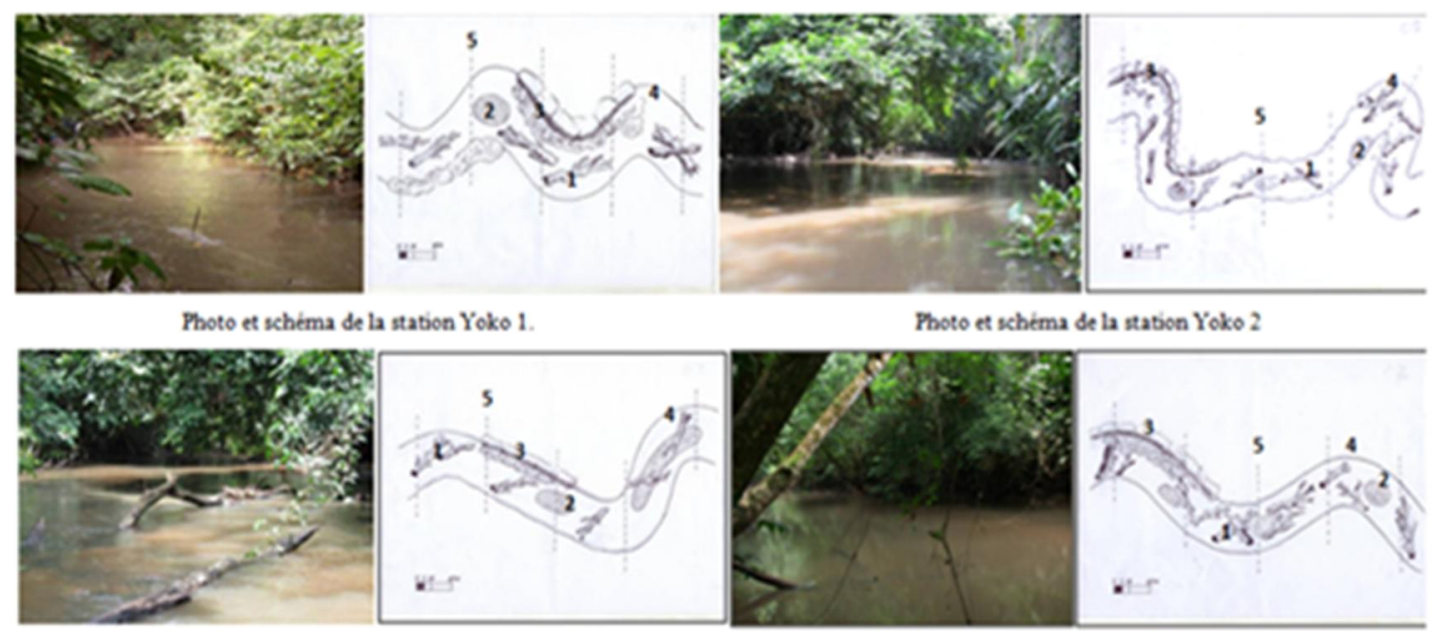

Photo et schéms de la station Yoko 3

Photo et schèms de la station Yoko 4
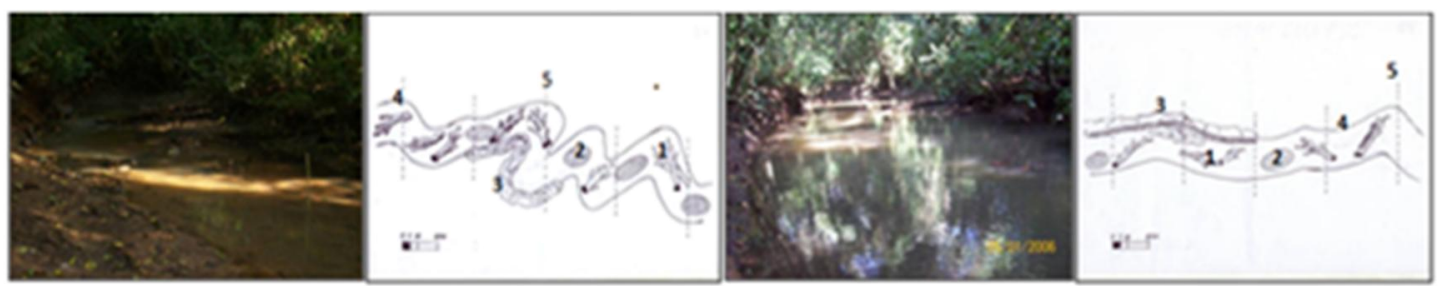

Photo et schema de la station Bure 2

Photo et schema de la station Biare 2

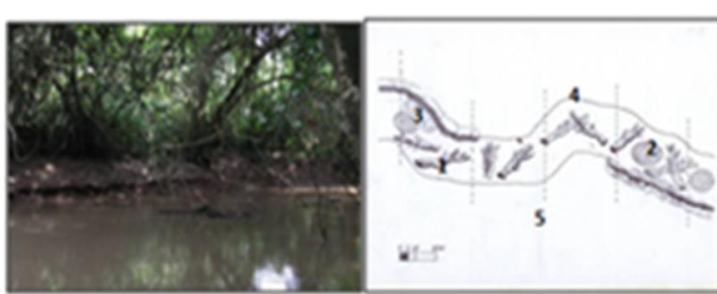

Photo et schèma de la station Biare 3

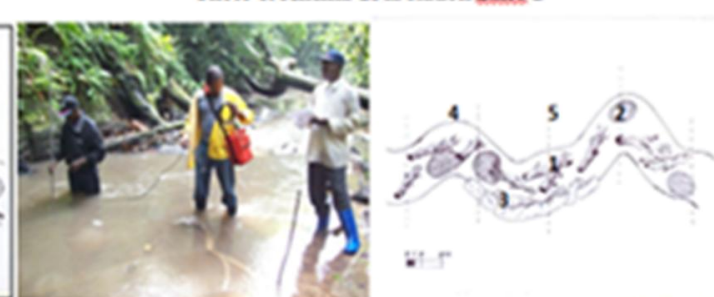

Photo et schèma de la station Bure 4 .

Figure 2 : Photos et schémas des stations montrant les ripisylves et types de substrats dans les 8 stations en rivières forestières Yoko et Biaro affluentes du fleuve Congo. 1 : Bois morts + branchages + débris végétaux $; 2:$ Bacs à sable + gravier + cailloux + galets + vases + limons $; 3:$ Berges à blocs de pierres et galet $; 4$ : Berges en argile + ripisylve ; 5 : Transect (profil en travers). 


\section{R. M. BYANIKIRO et al. / Int. J. Biol. Chem. Sci. 11(3): 967-999, 2017}

Tableau 1 : Caractéristiques hydromorphologiques et physico-chimiques moyennes comparées des huit stations des petites rivières Biaro et Yoko.

\begin{tabular}{|c|c|c|c|c|c|c|c|c|}
\hline Paramètres/Stations & Biaro 1 & Biaro 2 & Biaro 3 & Biaro 4 & Yoko 1 & Yoko 2 & Yoko 3 & Yoko 4 \\
\hline Largeur (m) & 6,69 & 17,85 & 18,32 & 8,00 & 8,14 & 14,77 & 11,09 & 15,76 \\
\hline Profondeur $(\mathrm{cm})$ & 28,47 & 66,90 & 70,30 & 42,06 & 20,17 & 38,16 & 29,42 & 47,95 \\
\hline Oxygène dissous (mg/l) & 5,16 & 5,55 & 5,25 & 5,56 & 4,80 & 5,21 & 5,47 & 5,20 \\
\hline Température $\left({ }^{\circ} \mathrm{C}\right)$ & 23,42 & 23,75 & 23,60 & 23,15 & 23,58 & 24,03 & 23,34 & 23,62 \\
\hline Saturation & 76,79 & 80,8 & 78,14 & 84,47 & 73,87 & 82,64 & 86,39 & 86,89 \\
\hline Salinité (ppm) & 46,58 & 38,58 & 40,04 & 43,23 & 61,06 & 54,83 & 43,35 & 47,83 \\
\hline Conductivité $\mathrm{K}_{20}(\mathrm{uS} / \mathrm{cm})$ & 91,26 & 86,61 & 82,95 & 88,85 & 121,04 & 108,67 & 90,72 & 93,51 \\
\hline $\mathrm{pH}$ & 7,96 & 7,87 & 8,02 & 7,87 & 7,97 & 8,13 & 7,57 & 7,99 \\
\hline Vitesse courant $(\mathrm{m} / \mathrm{sec})$ & 0,34 & 0,37 & 0,37 & 0,29 & 0,35 & 0,34 & 0,32 & 0,57 \\
\hline Transparence & Claire & claire & claire & Claire & claire & Claire & Claire & Claire \\
\hline Substrat & $\begin{array}{c}\text { Sablonneux, } \\
\text { rocheux et } \\
\text { caillouteux, } \\
\text { argileux et } \\
\text { limoneux; } \\
\text { branchages, } \\
\text { feuillages et } \\
\text { vases }\end{array}$ & $\begin{array}{c}\text { Sablo- } \\
\text { caillouteux, } \\
\text { galets et } \\
\text { graviers ; } \\
\text { branchages, } \\
\text { feuillages et } \\
\text { vases. }\end{array}$ & $\begin{array}{l}\text { Sablo-argileux, } \\
\text { rocheux avec } \\
\text { feuillages, bois } \\
\text { morts et débris } \\
\text { végétaux ; } \\
\text { vases et } \\
\text { limons. }\end{array}$ & $\begin{array}{l}\text { Sablonneux et } \\
\text { rocheux, } \\
\text { pierreux, } \\
\text { feuilles mortes, } \\
\text { bois morts et } \\
\text { débris } \\
\text { végétaux ; } \\
\text { vases et } \\
\text { limons. }\end{array}$ & $\begin{array}{c}\text { Sablonneux, } \\
\text { rocheux et } \\
\text { caillouteux } \\
\text { galets et } \\
\text { graviers ; } \\
\text { branchages, } \\
\text { feuillages ; } \\
\text { vases et } \\
\text { limons. }\end{array}$ & $\begin{array}{c}\text { Sablo- } \\
\text { caillouteux ; } \\
\text { galets et } \\
\text { graviers; } \\
\text { branchages, } \\
\text { feuillages et } \\
\text { vases. }\end{array}$ & $\begin{array}{c}\text { Sablonneux, } \\
\text { rocheux et } \\
\text { caillouteux } \\
\text { galets et } \\
\text { graviers ; } \\
\text { branchages, } \\
\text { feuillages ; } \\
\text { vases et } \\
\text { limons. }\end{array}$ & $\begin{array}{c}\text { Sablonneux ; } \\
\text { galets et } \\
\text { graviers ; } \\
\text { branchages, } \\
\text { feuillages et } \\
\text { vases et limons. }\end{array}$ \\
\hline
\end{tabular}


Tableau 2 : Familles et espèces végétales ripicoles inventoriées dans les huit stations des petites rivières Biaro et Yoko, affluentes du Congo.

\begin{tabular}{|c|c|c|c|c|c|c|c|c|c|}
\hline \multicolumn{2}{|c|}{ Végétation par strate } & \multicolumn{8}{|c|}{ Stations } \\
\hline \multirow{24}{*}{$\begin{array}{l}\text { Strate } \\
\text { supérieure }\end{array}$} & Familles et Espèces & Biaro 1 & Biaro 2 & Biaro 3 & Biaro 4 & Yoko 1 & Yoko 2 & Yoko 3 & Yoko 4 \\
\hline & Anacardiaceae & & & & & & & & \\
\hline & Pseudospondias microcarpa (A. R.) E. & + & + & + & + & + & & + & + \\
\hline & Annonaceae & & & & & & & & \\
\hline & Anonidium mannii (Oliver) Eng \& Diels & + & & & & + & & & \\
\hline & Cleistopholis patens Engler \& Diels & & + & + & + & & & & \\
\hline & Monodora myristica (Gaertner) Dunal & & & & & & & + & \\
\hline & Popowia bokoli (De W. \& th.) R. \& Gh & & + & & & & & & \\
\hline & Apocynaceae & & & & & & & & \\
\hline & Funtumia africana (Benth.) Stapf. & + & & + & & & & & \\
\hline & Landolphia owariensis P. Beauv. & + & + & + & & + & + & + & + \\
\hline & Arecaceae & & & & & & & & \\
\hline & Elaeis guineensis Jacq. & + & + & & & & & & \\
\hline & Eremospatha haullevilleana De Wil. & + & & + & + & + & + & & \\
\hline & Calamus deerratus Mann \& Wend. & & + & & & & & & \\
\hline & Raphia laurentii De Wild. & & + & + & & + & & & \\
\hline & Urticaceae & & & & & & & & \\
\hline & Musanga cecropioides $\mathrm{R}$. Br. & + & & + & & & & & + \\
\hline & Myrianthus arboreus P. Beauv. & + & + & + & & + & + & + & + \\
\hline & Myrianthus preussii Engler & + & + & + & + & & + & & \\
\hline & Clusiaceae & & & & & & & & \\
\hline & Allanblackia floribunda Oliver. & & & & & + & & & \\
\hline & Harungana madangascariensis L. Ex P. & & + & & & + & & & + \\
\hline & Ebenaceae & & & & & & & & \\
\hline
\end{tabular}


R. M. BYANIKIRO et al. / Int. J. Biol. Chem. Sci. 11(3): 967-999, 2017

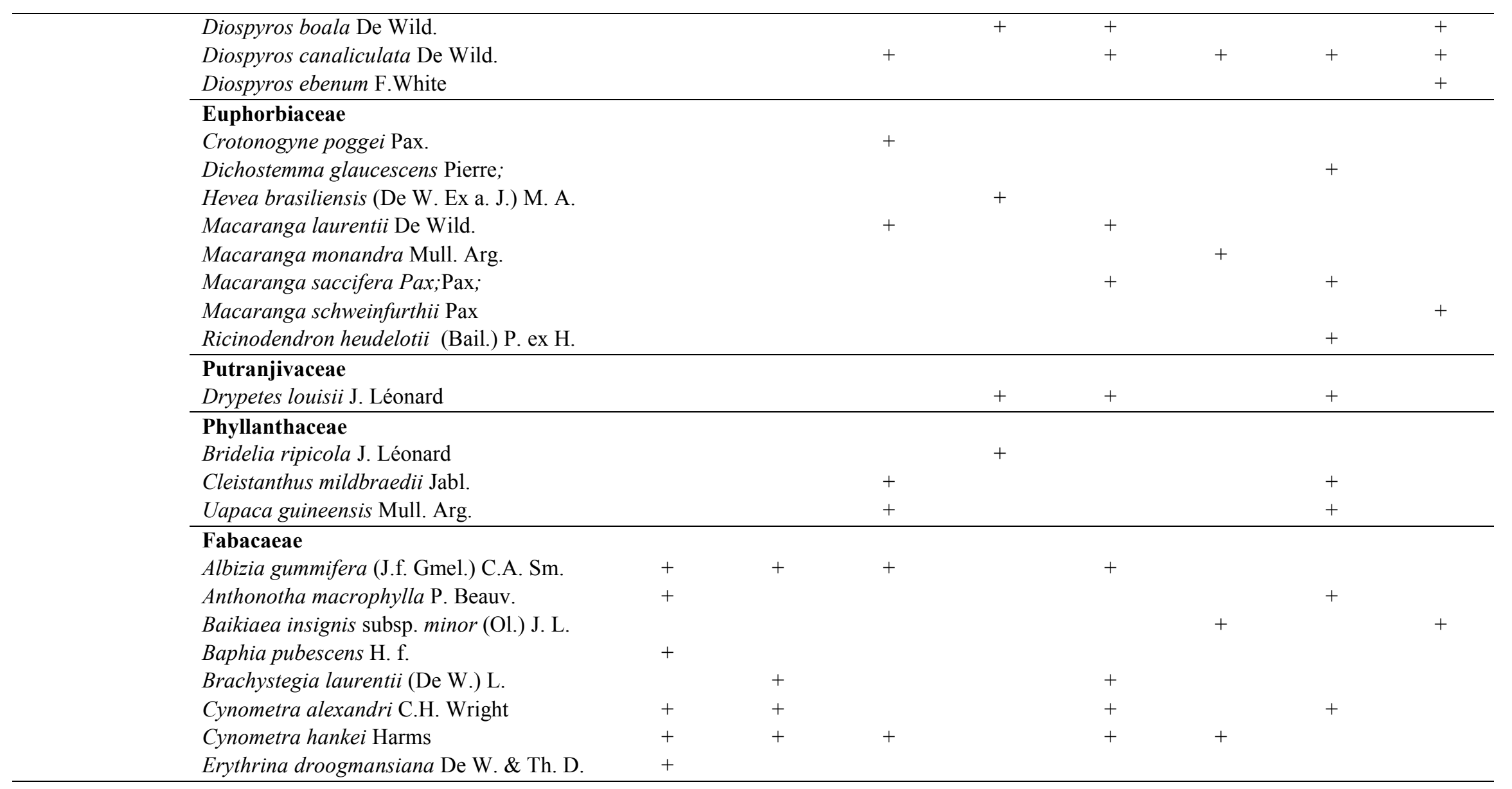


R. M. BYANIKIRO et al. / Int. J. Biol. Chem. Sci. 11(3): 967-999, 2017

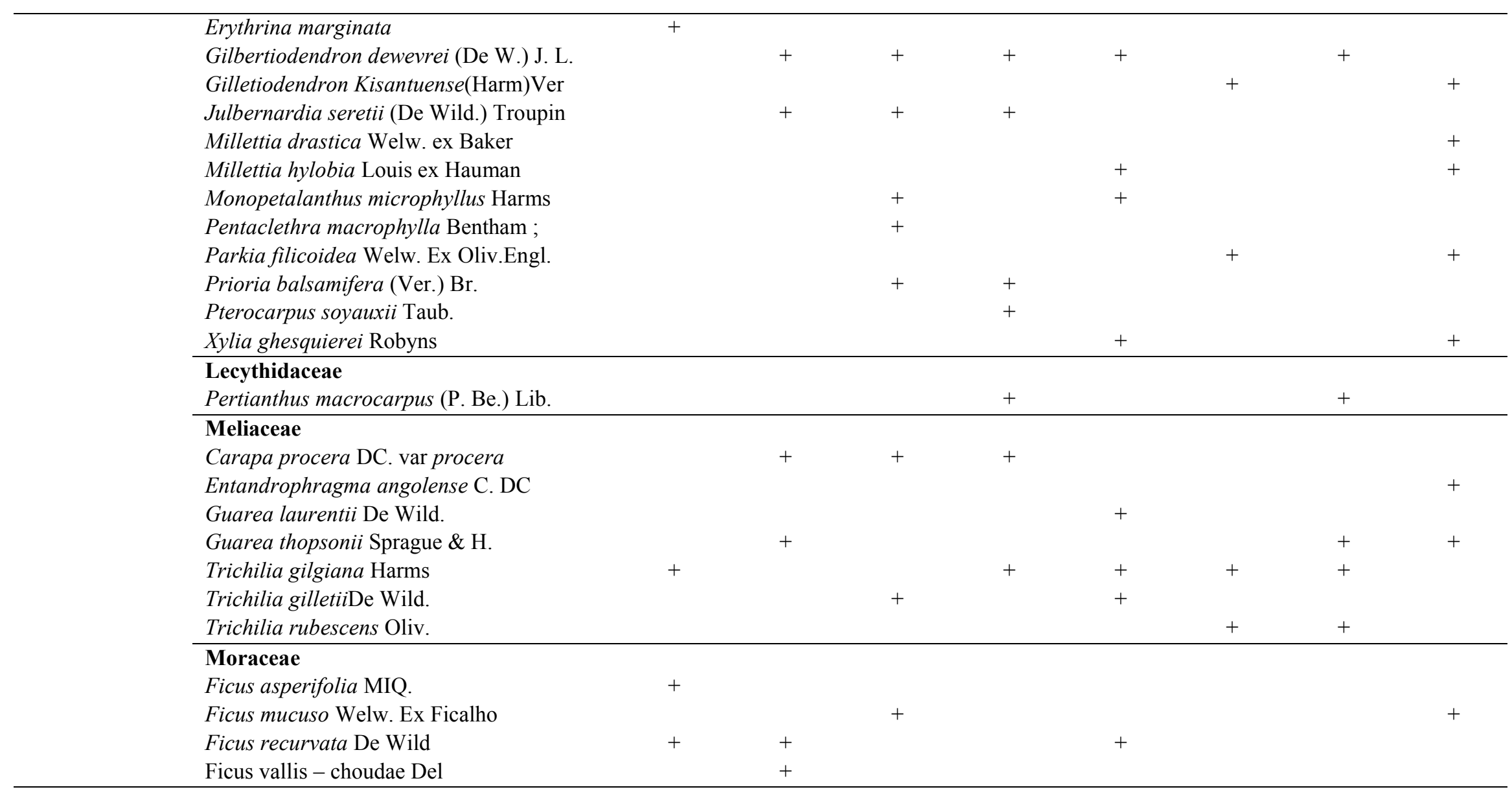


R. M. BYANIKIRO et al. / Int. J. Biol. Chem. Sci. 11(3): 967-999, 2017

\begin{tabular}{|c|c|c|c|c|c|c|c|c|}
\hline Ficus variifolia Warb. & & & & & & & & + \\
\hline Myristicaceae & & & & & & & & \\
\hline Pycnanthus angolensis (Welw.) Exell & + & + & + & + & & & & \\
\hline Pycnanthus marchalianus Ghesq. & & + & & & & & & + \\
\hline Strombosia nigropunctata Louis \& J. Lé. & & & + & & & + & & \\
\hline Strombosiopsis tetrandra Engler & & & + & & & & & \\
\hline Strombosia grandifolia HO. f. ex & & & & & & & & + \\
\hline BENTHAM & & & & & & & & \\
\hline Erythropalaceae & & & & & & & & \\
\hline Heisteria parvifolia Smith & & & & & & & + & \\
\hline Rubiaceae & & & & & & & & \\
\hline Cremaspora triflora (Thonn.) K. Schum. & & & & + & & & & \\
\hline Oxyanthus unilocularis Hiern & & + & & + & & & & \\
\hline Psychotria vogeliana Bentham & + & & + & + & & & & \\
\hline Psychotriagilgiana & & & & + & & & & \\
\hline Rutaceae & & & & & & & & \\
\hline Sapindaceae & & & & & & & & \\
\hline Blighia welwitschii (Hiern) Radlk. & & & & & & & + & \\
\hline Deinbollia longiacuminata Hauman & & & + & & & & & \\
\hline Eriocoelum microspermum Radlk. & & & + & & & & & + \\
\hline Sapotaceae & & & & & & & & \\
\hline Chrysophyllum africanum A. DC. & & & + & + & + & & + & + \\
\hline Pancovia harmsiana Gilg & + & & + & + & + & + & & \\
\hline Simaroubaceae & & & & & & & & \\
\hline Hannoa klaineana Pierre ex Engler] & & & + & & & & & \\
\hline
\end{tabular}


R. M. BYANIKIRO et al. / Int. J. Biol. Chem. Sci. 11(3): 967-999, 2017

\begin{tabular}{|c|c|c|c|c|c|c|c|c|}
\hline & Malvaceae & & & & & & & \\
\hline & Cola gigantea A. Chev. & & & & & & & + \\
\hline & Cola griseiflora De Wild & & & & & + & + & \\
\hline & Desplatsia dewevrei (De Wild. \& Th. Dur.) & & & + & & & + & + \\
\hline & Grewia trinervis De wild. & & & + & & & & \\
\hline & Leptonychia batangensis & & & + & & & & \\
\hline & Leptonychia multiflora K. Schum. & & & + & + & & & \\
\hline & Sterculia africana & & + & & & & & \\
\hline & Sterculia lwisii De Wild. & & + & & + & & + & \\
\hline & Sterculia tragacantha Lindley & & + & & & & & \\
\hline & Sterculia tragacantha Lindl & & & & & & & + \\
\hline & Irvingiaceae & & & & & & & \\
\hline & Irvingia grandifolia (Engler) Engler & & + & & & & & \\
\hline Strate & Anacardiaceae & & & & & & & \\
\hline intermédiaire & Lannea welwitschii (Hiern) Engler & & + & & & & & \\
\hline & Pseudospondias microcarpa (A. R.) E. & & & & & & & + \\
\hline & Artabotrys auriantiacus Engler \& Diels & + & + & & & + & & \\
\hline & Popowia bokoli (De W. \& th.) R. \& Gh & & + & + & & & + & \\
\hline & Arecaceae & & & & & & & \\
\hline & Calamus deeratus Mann.\& H. Wendl. & & + & + & + & + & & \\
\hline & Eremospatha haullevilleana De Wil. & & & & & & + & + \\
\hline & Elaeis guineensis Jacq. & & + & + & + & + & + & + \\
\hline & Raphia laurentii DE WILD. & & & + & & + & + & \\
\hline & Urticaceae & & & & & & & \\
\hline & Musanga cecropioides $\mathrm{R}$. Br. & & & & & & & + \\
\hline
\end{tabular}


R. M. BYANIKIRO et al. / Int. J. Biol. Chem. Sci. 11(3): 967-999, 2017

\begin{tabular}{|c|c|c|c|c|c|c|c|c|}
\hline Connaraceae & & & & & & & & \\
\hline Cnestis ferruginea DC & & + & & & & & & \\
\hline Cnestis urens Gilg & & + & & & & & & \\
\hline Costaceae & & & & & & & & \\
\hline Costus afer ker - Gawl. & + & & & & + & + & + & + \\
\hline Dichapetalaceae & & & & & & & & \\
\hline D. cfr mundense & & & & & + & & & \\
\hline Dichapetalum heudeloti (Pl. ex O.) Bail. & & & + & & + & + & + & + \\
\hline Dichapetalum lujaeTh. D. De W. var.lujae & & & & + & & & + & \\
\hline Dichapetalum mundense Engler & & & + & & & & & \\
\hline Dilleniaceae & & & & & & & & \\
\hline Tetracera alnifolia Willd & & + & + & & + & + & + & + \\
\hline Dioscoreaceae & & & & & & & & \\
\hline Dioscorea sp. & & & & & & & + & + \\
\hline Euphorbiaceae & & & & & & & & \\
\hline Crotonogyne poggei Pax. & + & & & + & & & & + \\
\hline Macaranga pynaertii De Wild; & + & & & & & & & \\
\hline Macaranga spinosa Mull. & & + & & & & & & \\
\hline Macaranga thonerii & & + & & & & & & \\
\hline Manihot esculenta Crantz & & & & & + & & & \\
\hline Manniophyton fulvum Mull. Arg. & + & + & & + & & + & + & + \\
\hline Macaranga saccifera Pax; & & + & & & & & & + \\
\hline Ricinodendron heudelotii (Bail.) P. ex H. & & & + & & & & & \\
\hline Manihot esculenta Crantz & & & & & & + & & \\
\hline Fabaceae & & & & & & & & \\
\hline Anthonotha macrophylla P. Beauv. & & & & & & & & + \\
\hline Anthonotha fragrans (Ba. f.) Ex. \& Hill. & & & & & & & & + \\
\hline
\end{tabular}


R. M. BYANIKIRO et al. / Int. J. Biol. Chem. Sci. 11(3): 967-999, 2017

\begin{tabular}{|c|c|c|c|c|c|c|c|c|}
\hline Brachystegia laurentii (De W.) L. & & & + & & + & & + & \\
\hline Baphia pubescens H. f. & & & & & & & & + \\
\hline Dewevrea bilabiata Micheli & & & + & + & & + & + & \\
\hline Dalbergia saxatilis Hooker var.saxatilis & + & & & & & & & \\
\hline Dalhousiea africana S. Moore & & & & + & & & + & \\
\hline Dialium excelsum Louis ex Steyaert & & & & & + & & & \\
\hline Gilbertiodendron dewevrei (De W.) J. L. & & & & & + & & & + \\
\hline Gilletiodendron Kisantuense (Harm) Ver. & & & & & & & + & \\
\hline Julbernardia seretii (De Wild.) Troupin & & & & & & & & + \\
\hline Millettia drastica Welw. ex Baker & & & & & & & & + \\
\hline Millettia duchesnei De Wild. & & + & + & + & + & + & + & \\
\hline Mucuna flagellipes Hooker f. & & & & & & & & \\
\hline Parkia filicoidea Welw. Ex Oliv. Engl. & & & + & + & & & & \\
\hline Tetrapleura tetraptera (Thonn.) Taub. & & & + & & & & & \\
\hline Salicaceae & & & & & & & & \\
\hline Barteria nigritana H.f. su. Fistulosa(M.) S. & & & & & & & + & + \\
\hline Caloncoba glauca (P. Beauv.) Gilg] & & & + & & & & & \\
\hline Caloncoba subtomentosa Gilg. & & & & + & & & & \\
\hline Hypolepidaceae & & & & & & & & \\
\hline Pteridium aquilinum (L.) Küuhn & & + & & + & & + & & \\
\hline Vitaceae & & & & & & & & \\
\hline Leea guineensis G. Don & + & + & + & + & & + & + & + \\
\hline Lomariopsidaceae & & & & & & & & \\
\hline Lomariopsis guineensis (Underw.) Alston & & & & + & & & & \\
\hline Meliaceae & & & & & & & & \\
\hline Carapa procera DC. var procera & + & & & & & & & \\
\hline Guarea thopsonii Sprague \& H. & & & & & & & & + \\
\hline
\end{tabular}


R. M. BYANIKIRO et al. / Int. J. Biol. Chem. Sci. 11(3): 967-999, 2017

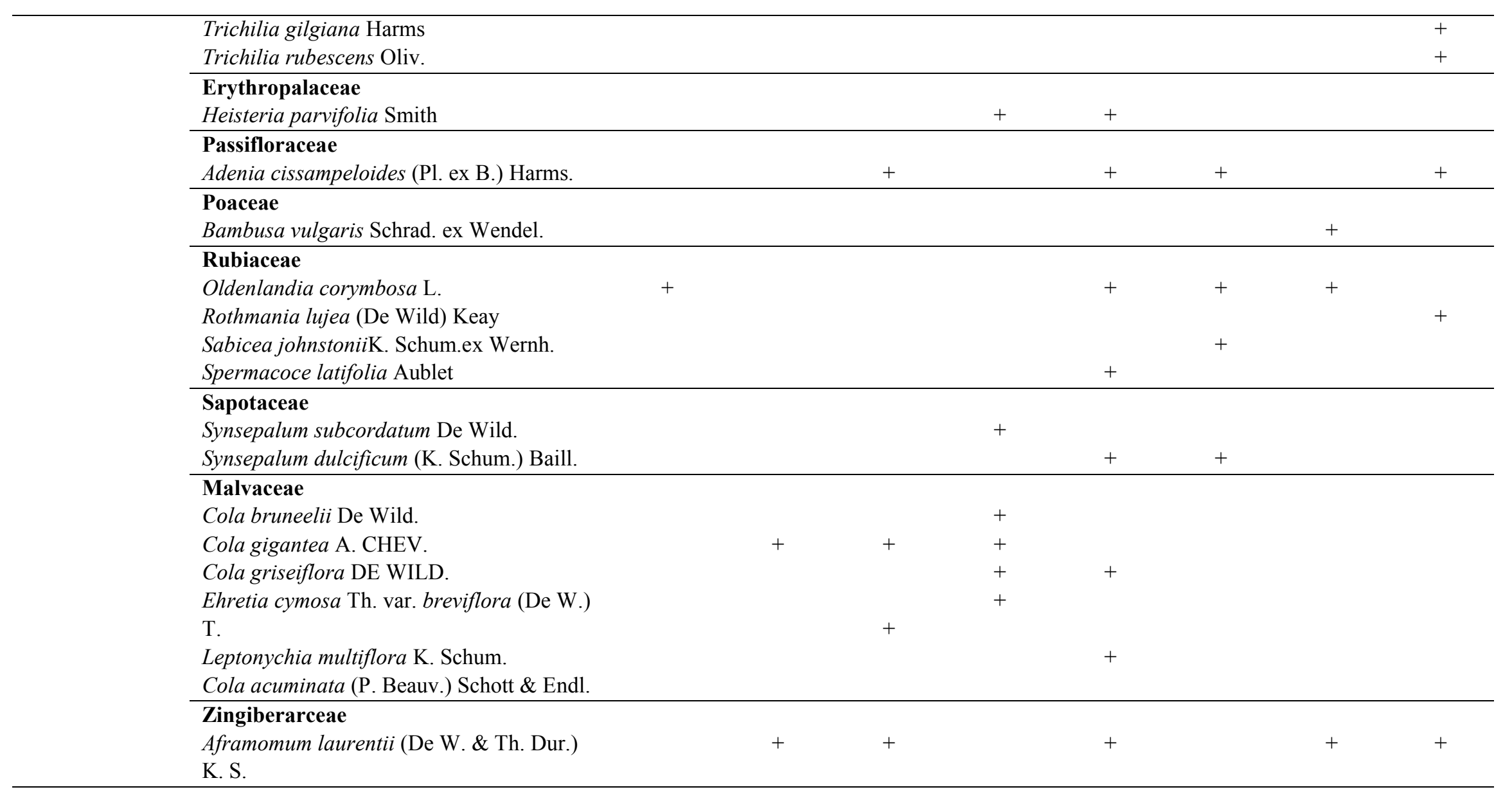


R. M. BYANIKIRO et al. / Int. J. Biol. Chem. Sci. 11(3): 967-999, 2017

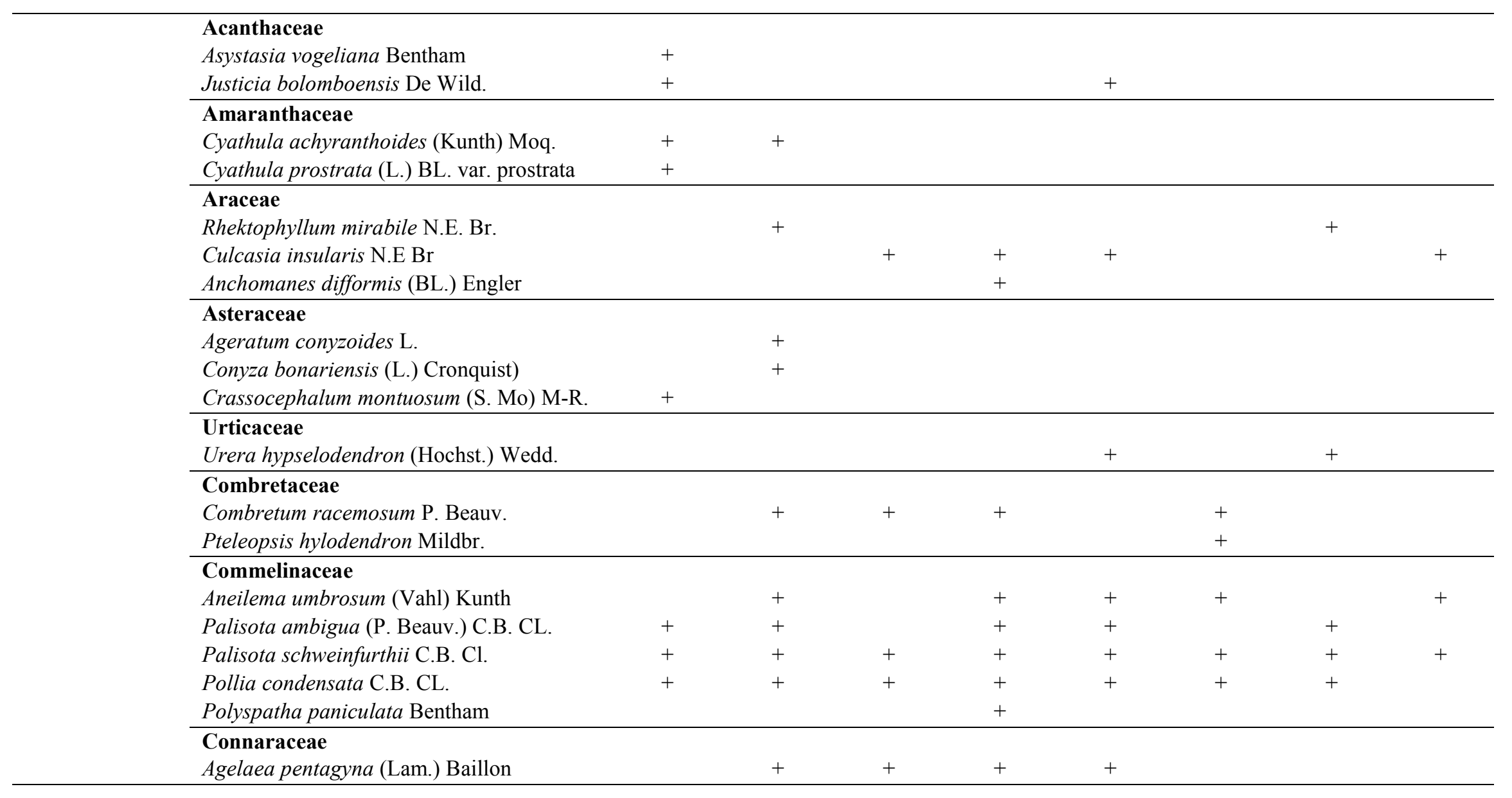


R. M. BYANIKIRO et al. / Int. J. Biol. Chem. Sci. 11(3): 967-999, 2017

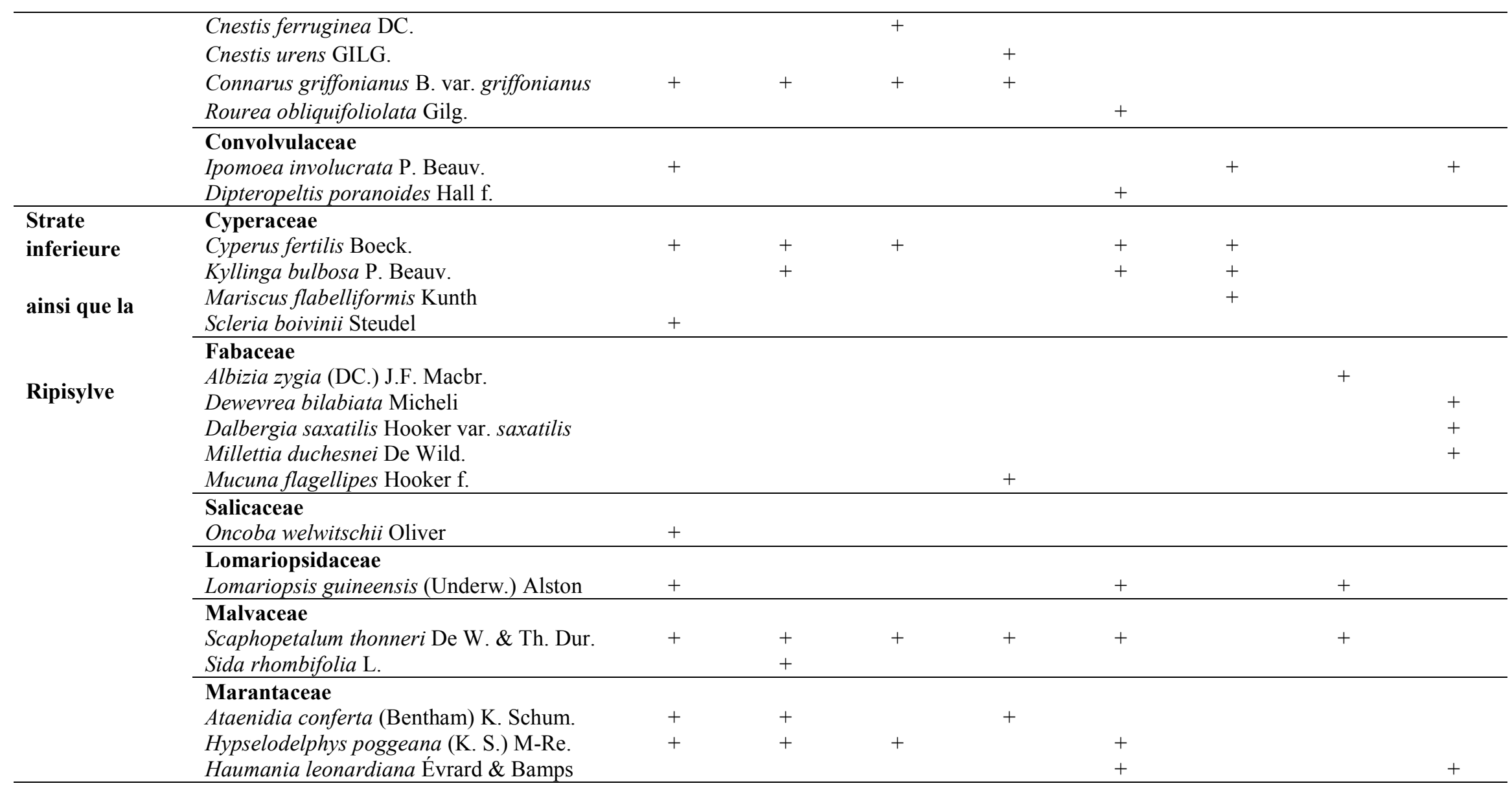


R. M. BYANIKIRO et al. / Int. J. Biol. Chem. Sci. 11(3): 967-999, 2017

\begin{tabular}{|c|c|c|c|c|c|c|c|}
\hline Hypselodelphys scandens Louis \& Mull. & & + & + & + & & & + \\
\hline Marantochloa sp. & + & + & & + & + & + & \\
\hline Megaphrynium macrostachyum (B.) M-Re. & + & + & + & + & & & + \\
\hline Sacrophrinium megalophrinium (B.) K. S. & & + & & & + & + & \\
\hline Sarcophrynium schweinfurthianum(k.)M- & & & + & & + & & \\
\hline $\begin{array}{l}\text { Re. } \\
\text { Thaumatococcus daniellii (Ben.) Be.\& H. }\end{array}$ & & & & + & + & + & + \\
\hline Melastomataceae & & & & & & & \\
\hline Tristemma mauritianum J.-F. Gmelin & & & & & + & & + \\
\hline $\begin{array}{l}\text { Kolobopetalum chevalieri (H. \& Dalz.) Tr. } \\
\text { Jateorhiza macrantha (H. f.) Exell \& Me. }\end{array}$ & & + & + & & & + & + \\
\hline $\begin{array}{l}\text { Primulaceae } \\
\text { Embellia guineensis Baker }\end{array}$ & & & & & + & & \\
\hline $\begin{array}{l}\text { Onagraceae } \\
\text { Ludwigia stolonifera (Guill. \& Perr.) Rav. }\end{array}$ & & & & + & + & + & + \\
\hline $\begin{array}{l}\text { Ochnaceae } \\
\text { Campylospermum elongatum (Oliver) Ti. }\end{array}$ & & & & + & & & \\
\hline $\begin{array}{l}\text { Phytolaccaceae } \\
\text { Hilleria latifolia (Lam.) Walter }\end{array}$ & & & & & & + & \\
\hline $\begin{array}{l}\text { Piperaceae } \\
\text { Piper umbellatum } \mathrm{L} .\end{array}$ & + & + & & & + & + & \\
\hline Poaceae & & & & & & & \\
\hline $\begin{array}{l}\text { Axonopus compressus (Sw.) P. Beauv. } \\
\text { Digitaria polybotrya } \text { Stapf; }\end{array}$ & $\begin{array}{l}+ \\
+\end{array}$ & $\begin{array}{l}+ \\
+\end{array}$ & + & & & & + \\
\hline Olyra latifolia L. & + & & & & + & + & + \\
\hline Panicum brevifolium L. & & + & & & + & & \\
\hline Paspalum conjugatum (Schultes) Berg. & & & & & & + & + \\
\hline Paspalum virgatum Steudel & + & + & & & & + & \\
\hline Setaria megaphylla (St.) Th. Dur. \& Schinz & & & + & & & & \\
\hline
\end{tabular}


R. M. BYANIKIRO et al. / Int. J. Biol. Chem. Sci. 11(3): 967-999, 2017

\begin{tabular}{|c|c|c|c|c|c|c|c|c|c|}
\hline & $\begin{array}{l}\text { Selaginellaceae } \\
\text { Selaginella myosurus (Sw.) Alston } \\
\text { Selaginella vogelii Spring }\end{array}$ & & + & & + & & + & + & \\
\hline & $\begin{array}{l}\text { Smilacaceae } \\
\text { Smilax anceps Willd }\end{array}$ & + & & + & + & & & + & + \\
\hline & $\begin{array}{l}\text { Solanaceae } \\
\text { Capsicum frutescens L. } \\
\text { Solanum americanum Miller; } \\
\text { Solanum torvum SW }\end{array}$ & & $\begin{array}{l}+ \\
+ \\
+\end{array}$ & & & & & & \\
\hline & $\begin{array}{l}\text { Thelypteridaceae } \\
\text { Christella dentata (Forsk.) Brownsey } \\
\text { Cyclosorus striatus (Schum.) Ching }\end{array}$ & & + & $\begin{array}{l}+ \\
+\end{array}$ & + & + & + & & + \\
\hline & $\begin{array}{l}\text { Cannabaceae } \\
\text { Celtis mildbraedii Engler } \\
\text { Trema orientalis }(\text { L.) Blume }\end{array}$ & & & & + & + & & & \\
\hline & $\begin{array}{l}\text { Vitaceae } \\
\text { Cayratia debilis (BAKER) Suesseng. } \\
\text { Cissus dinklagei Gilg \& Brandt. }\end{array}$ & & & & + & + & & & + \\
\hline Total par station & & $\begin{array}{l}\text { Familles } \\
: 27 \\
\text { Espèces : } \\
54\end{array}$ & $\begin{array}{l}\text { Familles } \\
\quad: 30 \\
\text { Espèces : } \\
\quad 78\end{array}$ & $\begin{array}{l}\text { Familles } \\
: 35 \\
\text { Espèces : } \\
74\end{array}$ & $\begin{array}{l}\text { Familles } \\
: 33 \\
\text { Espèces : } \\
68\end{array}$ & $\begin{array}{l}\text { Familles } \\
\quad: 36 \\
\text { Espèces : } \\
\quad 61\end{array}$ & $\begin{array}{l}\text { Familles } \\
\quad: 30 \\
\text { Espèces : } \\
52\end{array}$ & $\begin{array}{l}\text { Familles } \\
: 35 \\
\text { Espèces : } \\
63\end{array}$ & $\begin{array}{l}\text { Familles } \\
\quad: 28 \\
\text { Espèces } \\
\quad: 59\end{array}$ \\
\hline
\end{tabular}

Légende :+ = Présence

Total Général des familles $=\mathbf{5 6}$ et total général des espèces $=\mathbf{1 9 5}$ 
Tableau 3 : Liste systématique, effectifs et abondances relatives des Mormyridae dans les 8 stations des petites rivières forestières Biaro et Yoko, bassin du Congo.

\begin{tabular}{lllcc}
\hline Familles & Genres & Espèces & Effectifs & $\begin{array}{c}\text { Abondance } \\
\text { relative (\%) }\end{array}$ \\
\hline MORM & CyphomyrusMyers, 1960 & Cyphomyrus psittacus Boulenger, 1897 & 2 & 0,9 \\
RIDAE & MarcuseniusGill, 1862 & Marcusenius brucii Boulenger, 1910 & 2 & 0,9 \\
& & Marcusenius cyprinoidesLinnaeus, 1758 & 9 & 4,2 \\
& & Marcusenius senegalensisSteindachner, 1870 & 3 & 1,4 \\
& PetrocephalusMarcusen, & Petrocephalus microphtalmus Pellegrin, 1908 & 50 & 23,4 \\
& 1854 & Petrocephalus pallidomaculatusBogome et & 4 & 1,9 \\
& & Paugy, 1991 & & \\
& StomatorhinusBoulenger, & Stomatorhinus cornetiBoulanger, 1899 & 144 & 67,3 \\
& 1898 & & & $\mathbf{2 1 4}$ \\
\hline TOTAL & $\mathbf{4}$ & $\mathbf{7}$ & $\mathbf{1 0 0 , 0}$ \\
\hline
\end{tabular}

Tableau 4 : Effectifs totaux et pourcentages stationnels des Mormyridae capturés dans les 8 stations des petites rivières forestières Biaro et Yoko, bassin du Congo.

\begin{tabular}{|c|c|c|c|c|c|c|c|c|c|}
\hline Especes & Biaro 1 & Biaro 2 & $\begin{array}{c}\text { Biaro } \\
3\end{array}$ & Biaro 4 & Yoko 1 & Yoko 2 & Yoko 3 & Yoko 4 & Total \\
\hline Cyphomyrus psittacus & & & 1 & & & 0 & 0 & 1 & 2 \\
\hline Marcusenius brucii & 0 & 0 & 0 & 0 & 0 & 0 & 0 & 2 & 2 \\
\hline Marcusenius cyprinoides & 0 & 1 & 2 & 0 & 2 & 0 & 0 & 4 & 9 \\
\hline Marcusenius senegalensis & 0 & 0 & 0 & 0 & 0 & 0 & 0 & 3 & 3 \\
\hline $\begin{array}{l}\text { Petrocephalus } \\
\text { microphtalmus }\end{array}$ & 9 & 16 & 10 & 7 & 0 & 0 & 0 & 8 & 50 \\
\hline $\begin{array}{l}\text { Petrocephalus } \\
\text { pallidomaculatus }\end{array}$ & 0 & 0 & 4 & 0 & 0 & 0 & 0 & 0 & 4 \\
\hline Stomatorhinus corneti & 51 & 49 & 18 & 11 & 0 & 0 & 0 & 15 & 144 \\
\hline $\begin{array}{l}\text { Effectifs totaux par } \\
\text { station }\end{array}$ & 60 & 66 & 35 & 18 & 2 & 0 & 0 & 33 & 214 \\
\hline Pourcentage (\%) & 28,04 & 30,84 & 16,35 & 8,41 & 0,93 & $\mathbf{0}$ & $\mathbf{0}$ & 15,43 & 100,00 \\
\hline
\end{tabular}

Tableau 5 : Effectifs totaux, indices de diversité (H') de Shannon, de Simpson (1-D) et équitabilité (E des populations de Mormyridae dans les huit stations des petites rivières forestières Biaro et Yoko.

\begin{tabular}{lcccccccc}
\hline Indices / Stations & Biaro1 & Biaro2 & Biaro3 & Biaro4 & Yoko1 & Yoko2 & Yoko3 & Yoko4 \\
\hline Taxa (N) & 2 & 3 & 5 & 2 & 1 & 0 & 0 & 6 \\
Individus (N) & $\mathbf{6 0}$ & $\mathbf{6 6}$ & $\mathbf{3 5}$ & $\mathbf{1 8}$ & $\mathbf{2}$ & $\mathbf{0}$ & $\mathbf{0}$ & $\mathbf{3 3}$ \\
Shannon (H') & 0,423 & 0,628 & 1,213 & 0,668 & 0 & $* * * *$ & $* * * *$ & 1,452 \\
Simpson (1-D) & 0,255 & 0,39 & 0,637 & 0,475 & 0 & $* * * *$ & $* * * *$ & 0,707 \\
Equitabilité (E) & 0,61 & 0,572 & 0,754 & 0,964 & 0 & $* * * *$ & $* * * *$ & 0,81 \\
\hline
\end{tabular}

990 
Tableau 6 : Indice de similarité entre les stations et entre les espèces de Mormyridae des 8 stations des petites rivières forestières Biaro et Yoko.

\begin{tabular}{|c|c|c|c|c|c|c|}
\hline \multicolumn{4}{|c|}{ Similarité entre les stations } & \multicolumn{3}{|l|}{ Similarité entre les espèces } \\
\hline & Axis 1 & Axis 2 & Axis 3 & & Axis 1 & Axis 2 \\
\hline Eigenvalues & 1,235 & 0,615 & 0,437 & Eigenvalues & 1,098 & 0,285 \\
\hline Pourcentage & 47,27 & 23,521 & 16,706 & Pourcentage & 62,651 & 16,233 \\
\hline Pourcentage Cumulé & 47,27 & 70,791 & 87,497 & Pourcentage Cumulé & 62,651 & 78,884 \\
\hline \multirow[t]{2}{*}{ PCO case scores } & & & & PCO case scores & & \\
\hline & Axis 1 & Axis 2 & Axis 3 & & & \\
\hline Biaro 1 & $-0,096$ & 0,368 & $-0,182$ & & Axis 1 & Axis 2 \\
\hline Biaro 2 & 0,031 & 0,388 & $-0,092$ & Cyphomyrus psittacus & $-0,257$ & $-0,105$ \\
\hline Biaro 3 & 0,485 & 0,133 & 0,514 & Marcusenius brucii & $-0,274$ & $-0,134$ \\
\hline Biaro 4 & $-0,133$ & 0,158 & $-0,12$ & Marcusenius cyprinoides & $-0,213$ & 0,479 \\
\hline Yoko 1 & $-0,238$ & $-0,283$ & 0,154 & Marcusenius senegalensis & $-0,26$ & $-0,108$ \\
\hline Yoko 3 & $-0,407$ & $-0,212$ & 0,015 & Petrocephalus microphtalmus & 0,462 & 0,016 \\
\hline Yoko 2 & $-0,407$ & $-0,212$ & 0,015 & Petrocephalus pallidomaculatus & $-0,221$ & $-0,118$ \\
\hline Yoko 4 & 0,764 & $-0,341$ & $-0,303$ & Stomatorhinus corneti & 0,763 & $-0,03$ \\
\hline
\end{tabular}
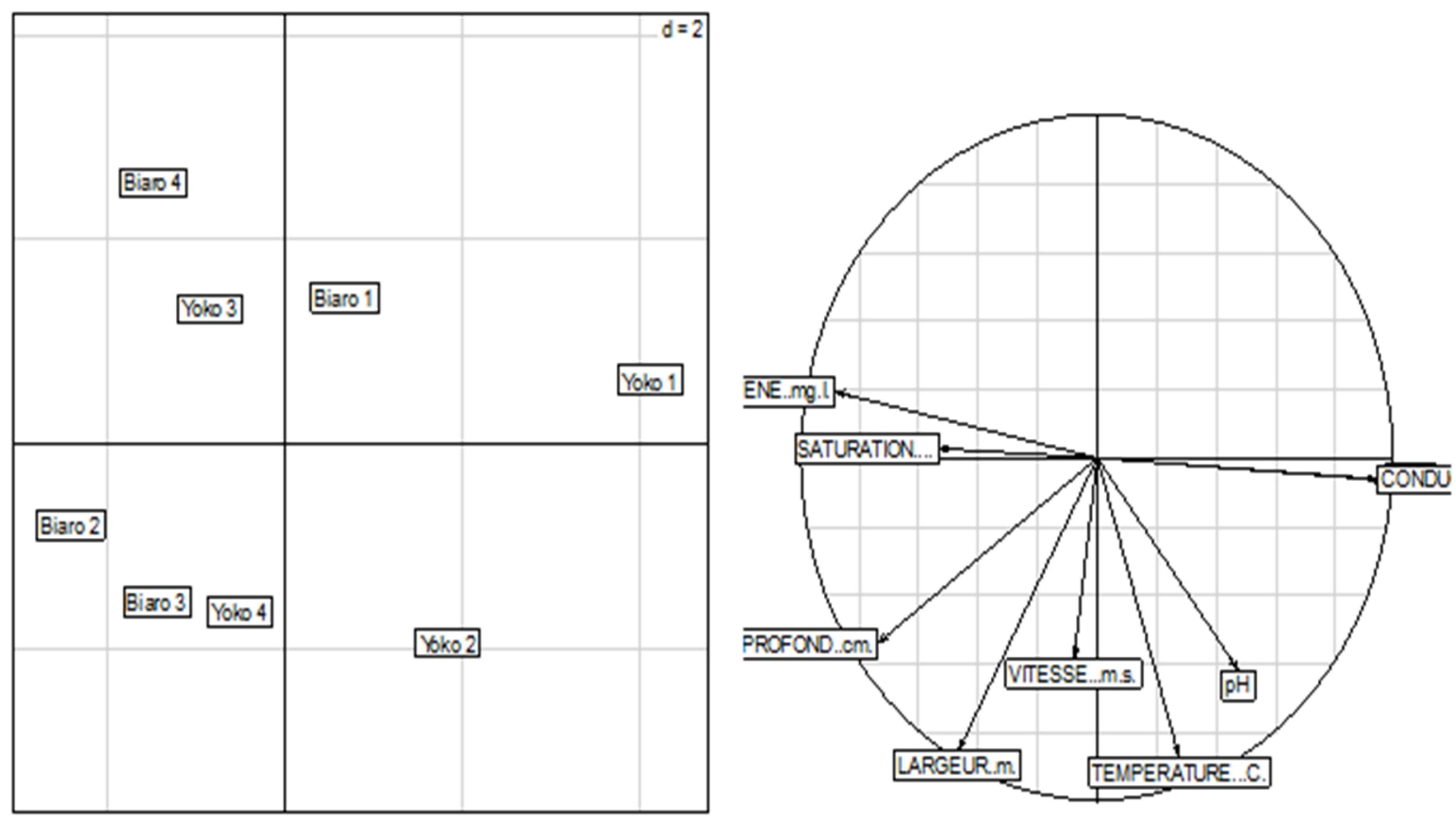

Figure 3 : Résultats des Analyses en Composantes Principales (ACP) entre les stations et les paramètres hydromorphologiques et physico-chimiques des 8 stations des rivières Yoko et Birao, affluentes du fleuve Congo. 


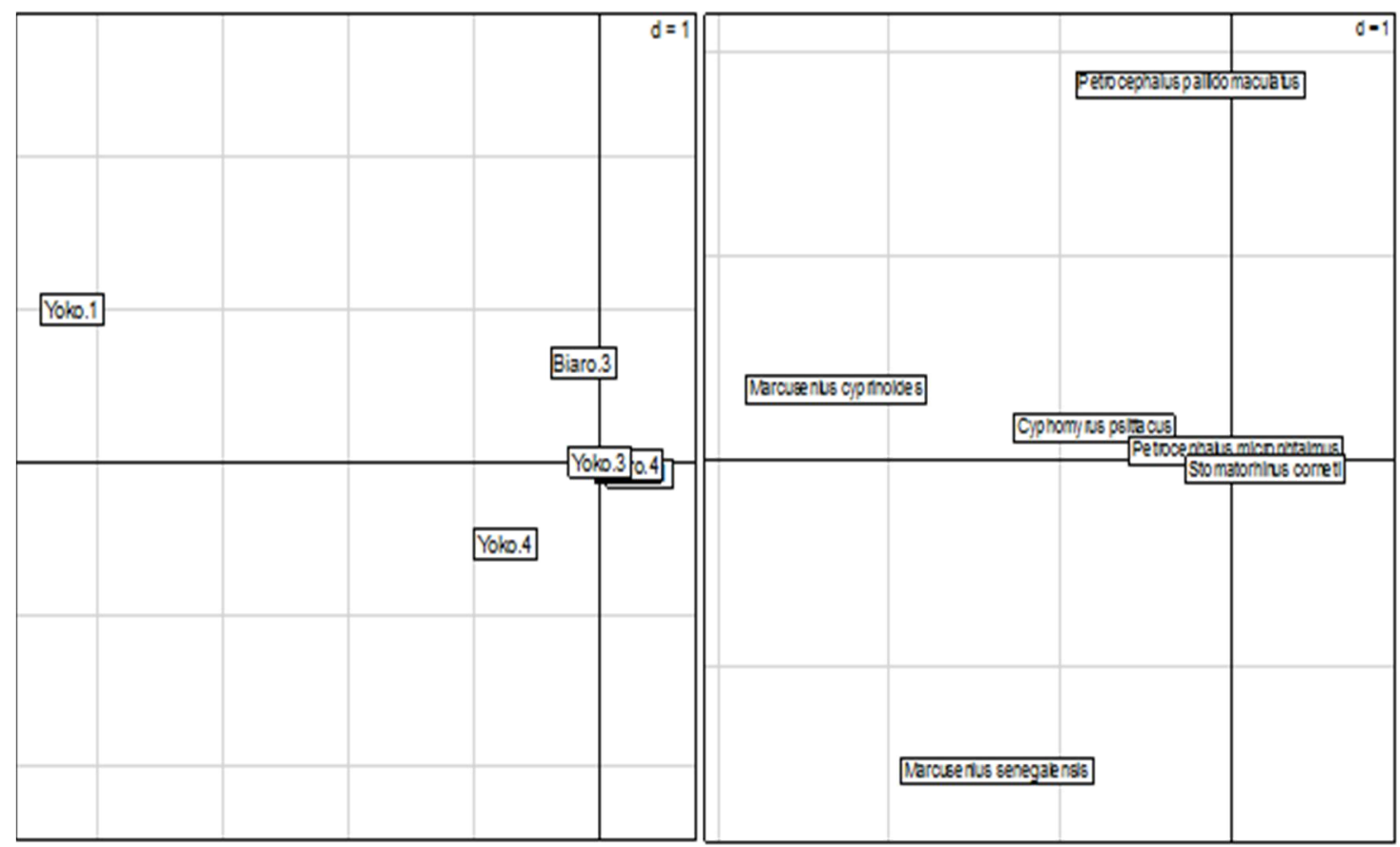

Figure 4 : Résultats des Analyses Factorielles de Correspondance (AFC) sur la réparation spatiale des espèces de Mormyridae (septembre 2008- août 2009) dans 8 stations des rivières Yoko et Biaro, affluentes du fleuve Congo.

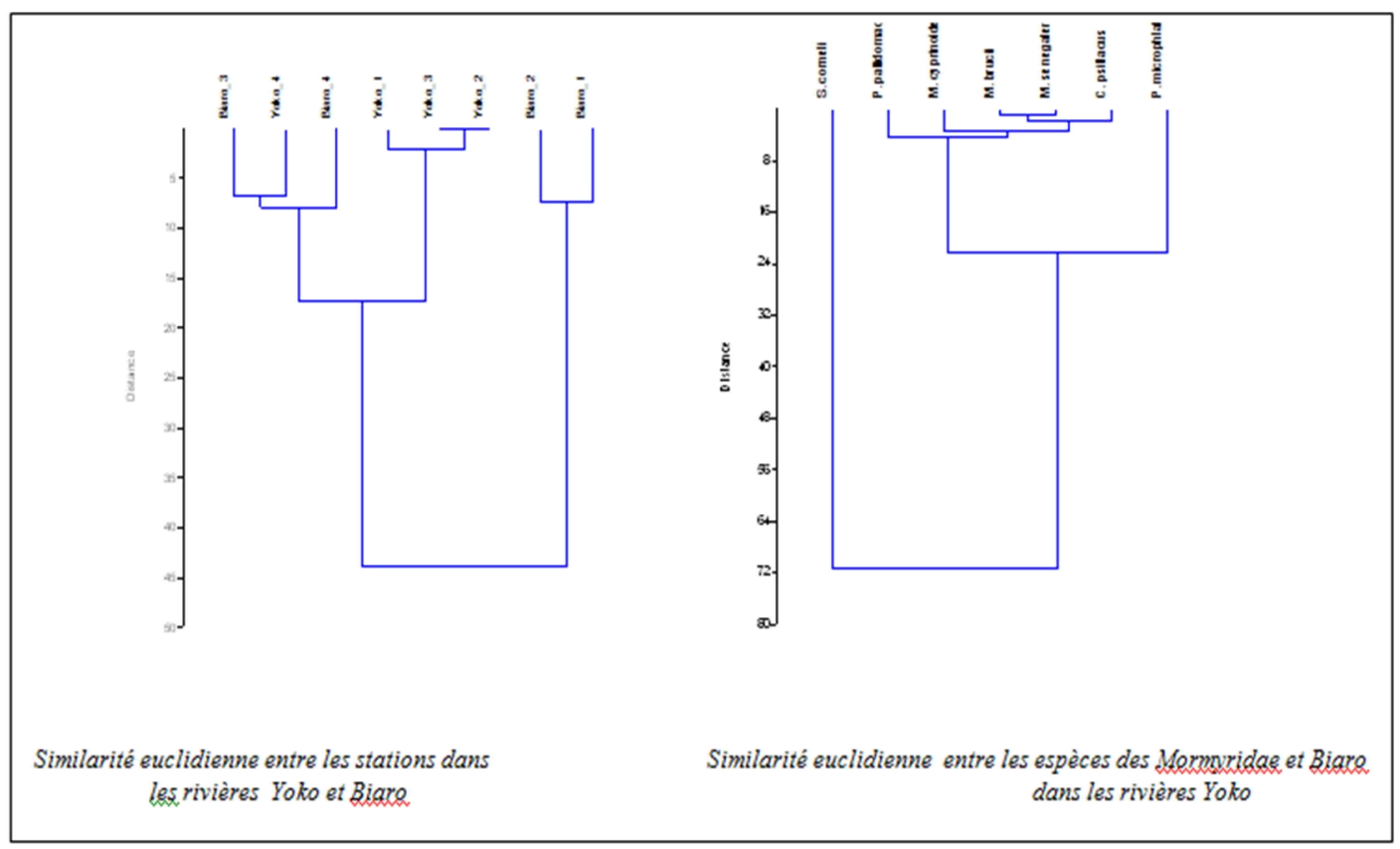

Figure 5: Dendrogramme de similarité euclidienne entre les espèces de Mormyridae dans les rivières Yoko et Biaro, affluentes du fleuve Congo. 


\section{DISCUSSION}

\section{Caractérisation des stations et distribution des espèces de Mormyridae}

Les stations d'échantillonnage ont été comparées entre elles à partir de chaque paramètre hydromorphologique mesuré mensuellement, indépendamment des autres facteurs pendant chaque mois de récolte (Tableau 1). La variation de la largeur des rivières et leur profondeur changent de l'amont en aval et d'une rivière à une autre. Ainsi, les stations Biaro 3 et 2, Yoko 4 et 2 présentent de plus grandes valeurs de largeur et de profondeur qui contribuent à une plus grande diversité ichtyologique. Plusieurs études réalisées sur différents hydrosystèmes ont déjà établi des relations entre espèces et variables environnementales (Kouamélan et al., 2003 ;Yao et al., 2005 ; Kouadio et al., 2006 ; Kouamé et al., 2008). Chacune de ces variables joue directement ou indirectement un rôle important sur la faune ichtyologique.

La température moyenne de l'eau dans un cours d'eau de la forêt tropicale dépasse rarement $24{ }^{\circ} \mathrm{C}$ comme c'est le cas pour les stations de recherche pour cette étude, ceci est dû, d'une part, à la pluviosité élevée dans cette forêt qui est supérieure à $1600 \mathrm{~mm} / \mathrm{an}$ et d'autre part à l'ombrage causé par des cimes d'arbres contigües dans la forêt ombrophile équatoriale. Ceci favorise le maintien d'une fraîcheur des eaux qui s'écoulent et crée des conditions stables, favorables aux organismes qui y vivent notamment les poissons. Kankonda (2007) travaillant dans le ruisseau Masangamabe $\left(24,75\right.$ à $\left.25,92{ }^{\circ} \mathrm{C}\right)$ a fait les mêmes observations. Da Costa et al. (2000), de leur côté, en Côte d'Ivoire, mentionnent qu'une amplitude faible de variation de la température moyenne de l'eau peut avoir une influence sur l'abondance et la répartition temporelle des espèces de poissons.

Les concentrations moyennes annuelles d'oxygène dissous pour l'ensemble de 8 stations prospectées sont relativement bonnes $(4,8$ à $5,6 \mathrm{mg} / \mathrm{l})$ avec des pourcentages de saturation d'oxygène de 73,9 à 86,9 . Ce léger déficit de saturation résulte probablement de la décomposition presque continue des matières organiques (feuilles mortes, débris végétaux et animaux, graines, fruits, etc.) par les décomposeurs (bactéries et champignons). Da Costa et al. (2000); Aboua et al. (2010), travaillant dans différents sites en Côte d'Ivoire, ont montré l'importance de ces variables dans la distribution des poissons. Les résultats de cette étude abondent dans le même sens que ceux de ces auteurs tant bien même qu'elle soit menée dans deux petits cours d'eau forestiers en pleine réserve de Yoko et où aucune étude de ce genre n'y a jamais été effectuée. Nous pensons que les résultats de ce travail contribueront à la bonne gestion de cet écosystème.

La vitesse du courant est sans conteste, un des paramètres essentiels à la base de la répartition des populations végétales et animales (Verniers, 2005). Elle dépend de la pente, de la rugosité des berges et du lit. Les moyennes annuelles de la vitesse du courant d'eau pour cette étude ont varié de 0,29 à 0,57 $\mathrm{m} / \mathrm{s}$, ce qui correspond à un courant moyen suivant l'échelle de classification générale du courant d'eau proposée par Verniers (2005). Cette affirmation confirme les observations de Lemoalle (2006) qui dit que, dans la plupart des cours d'eau, de la source à l'estuaire, on observe un changement progressif de l'altitude, de la pente, de la vitesse du courant et de la section (largeur et profondeur) de la rivière. Ces conditions de milieu déterminent des biotopes successifs, depuis le petit torrent sur substrat rocheux jusqu'au large fleuve aux méandres envasés, auxquels correspondent des peuplements également bien différenciés.

Les différentes stations de recherche font état d'une grande variabilité de par la composition et la structure des éléments (nature des berges, nature des fonds, ripisylve et végétation forestière) qui les constituent dans des proportions différentes (Tableau 1 et 2). Cette végétation, outre son rôle écologique (création de microhabitats, modification de la texture du sol, maintien des paramètres physicochimiques, modification du substrat, etc.) est aussi source d'aliment car certaines parties d'espèces végétales comme les feuilles, fruits qui tombent dans l'eau sont 
immédiatement consommées par les poissons, par exemple: Pseudopondias microcarpa, Anonidium mannii, Myrianthus arboreus, Uapaca guinnensis etc. De plus, troncs d'arbres morts, feuilles mortes, fruits pourris immergés dans l'eau fournissent des oligochètes, des larves d'insectes tels que les Chironomidae aux poissons. Cela va dans le même sens que les observations d'Aboua et al. (2010).

Selon Ashley et al. (2008), dans l'écorégion 539, les rivières forestières tropicales à plaines inondables sont constituées de microhabitats variés, susceptibles d'être la source d'une grande biodiversité et d'endémismes. On pense que le nombre d'espèces des Mormyridae inventoriées (7 espèces) résulte de ce facteur. Selon Alhou (2007), les résultats de diversité pourraient être influencés par des facteurs comme la diversité d'habitats. Ce même auteur précise que la diversité des substrats permet un échantillonnage plus riche car ils constituent des micros habitats supplémentaires induits dans les stations d'échantillonnages qui sont susceptibles d'améliorer la diversité taxonomique. En attendant, on doit se contenter des variables hydrologiques classiques (nature de fonds, de berges, largeur du lit, profondeur, etc.).

En faisant allusion aux résultats des Analyses en Composantes Principales (Figure 3), on constate qu'il existe quatre entités écologiques où, d'une part, Yoko 1 et Biaro 1 ont tendance à se ressembler, grâce à la végétation et la nature du substrat, d'autre part, Biaro 2, Biaro 3, et Yoko 4 se ressemblent par la profondeur, la largeur, ainsi que la vitesse de l'eau, alors que Yoko 4 et Biaro 3, situées à l'aval en pleine réserve de la Yoko où les conditions écologiques sont plus stables, présentent le même niveau de saturation en oxygène dissous. C'est pourquoi, les stations Biaro 4 et Yoko 3 constituant un même pool écologique enregistrent respectivement 2 et 6 espèces de Mormyridae recherchant la saturation en oxygène de l'eau ; tandis que les espèces rencontrées dans les stations Biaro 2 et 3 et Yoko 4 sont plutôt corrélées à la profondeur, la largeur, la vitesse de l'eau ainsi qu'à la végétation. On constate aussi une augmentation du nombre d'espèces le long du gradient amont-aval par l'addition successive d'espèces plutôt que par un remplacement de celles-ci comme le confirment (Hugueny et Lévêque, 2006; Aboua et al., 2010). Les différences des données observées au niveau des stations pourraient provenir, d'après Kouamélan (2003) des méthodes de pêches utilisées, des types d'habitats échantillonnés et de la période d'échantillonnage. Cette disposition obéit aux observations relatives de la zonation longitudinale des cours d'eau africains énoncé par Lévèque et Paugy (2006) et confirmé par Kouamé et al. (2008) sur le fleuve Sassandra en Côte d'Ivoire ainsi que par Aboua et al. (2010).

Dans le cours d'eau principal, les associations ichtyologiques sont en conformité avec les zonations longitudinales bien connues (Lévèque et Paugy, 2006), montrant une augmentation progressive de la richesse spécifique de l'amont vers l'aval (Lalèyè et al., 2004; Lévèque et Paugy, 2006 ; Aboua et al., 2010). Par contre, Yoko 2, sans Mormyridae, ne présente aucune similarité avec d'autres stations et se caractérise par une végétation très jeune due au fait que, cette station se situe en dehors de la réserve, à proximité de la route Kisangani Ubundu et est donc soumise à de fortes activités anthropiques (champs, bois de chauffe, piègeage, écopage, etc.) résultant en jachères et recrus forestiers. Néanmoins, des altérations peuvent apparaître dans certaines parties du cours d'eau, dues surtout aux activités anthropiques et traduire en conséquence une certaine réduction de la richesse spécifique avec une disparition des espèces sensibles (Lévêque et Mounolou, 2008 ; Aboua et al., 2010, Kerckhove, 2012). Qu'il s'agisse d'eau courante ou stagnante, la situation géographique d'un système gouverne, indirectement mais assez exactement, la qualité physico-chimique et hydromorphologique du milieu, par un ensemble complexe d'interrelations entre les 
différentes variables qui décrivent l'environnement. Les conditions abiotiques et biotiques $\mathrm{du}$ milieu influent directement sur les communautés de poissons (oxygène dissous et température, salinité et transparence, nature de la ripisylve, couvert forestier, etc.).

\section{Caractérisation et composition faunistique des peuplements de Mormyridae.}

Conformément à la littérature Lalèyè et al. (2004), Aboua et al. (2010), la famille des Mormyridae domine dans les cours d'eaux africains en matière de diversité spécifique et ici plus particulièrement dans la partie amont des petits cours d'eau affluents du fleuve Congo. Il est probable qu'on n'ait pas capturé toutes les espèces présentes. Cependant, on a constaté que la pêche aux filets maillants et la pêche à la ligne ne donnent pas de très bons résultats dans ces petits cours d'eau. D'après Kouamélan (2003) cela est dû aux méthodes de pêches utilisées, aux types d'habitats échantillonnés et à la période d'échantillonnage. La pêche à la nasse semble la plus appropriée et serait idéalement complétée par d'autres méthodes comme la pêche à l'épuisette. Cette dernière fournit essentiellement de petits spécimens à la diversité importante. Cette affirmation est confirmée par Pigneur (2005). Dans les petits affluents du fleuve Congo, la richesse taxonomique la plus élevée est observée aux stations Yoko 4 et Biaro 3 avec respectivement 6 et 5 espèces de Mormyridae, ce qui correspond aussi à une plus grande diversité d'habitats (zones lotiques et lentiques). Selon Lévèque et Paugy (2006) ; Aboua et al. (2010), la richesse spécifique d'un milieu traduit sa capacité d'accueil qui est d'autant plus grande que le nombre de niches écologiques occupées est élevé.

Distribution spatiale des espèces de Mormyridae dans les différentes stations

Les résultats des AFC (Figure 4) sur la répartition spatiale des espèces capturées par station et leurs effectifs (Tableau 3) montrent clairement qu'il existe des liens entre les stations et les espèces comme détailler plus haut et confirmés par les espèces telles que $S$. corneti, P. microphtalmus qui sont fortement associées aux stations Yoko 2 et 3, Biaro 1, 2 et 4 suite à la corrélation positive observée entre les variables (végétation, température de l'eau, vitesse de l'eau, profondeur de l'eau, oxygène dissous et nature des substrats). Ces liens entre les espèces et les stations, s'expliqueraient par des préférences particulières de chaque espèce à des microhabitats où elles trouvent réunies les meilleures conditions pour leur survie. Ceci confirme l'hypothèse selon laquelle, la forêt tropicale humide, source d'hétérogénéité d'habitats des rivières qui y coulent, influerait sur la structure spatio-temporelle des peuplements des Mormyridae.

Selon Lemoalle (2006), une espèce de poisson se maintient à long terme dans un hydrosystème dans la mesure où elle y trouve un ensemble de conditions qui lui permettent, entre autres, de croître et de se reproduire. Ces conditions se répartissent schématiquement en deux ensembles : ll'environnement physicochimique (ou abiotique), constitué par les contextes géologique et climatique et le paysage aquatique tel qu'il est perçu par le poisson ; l'environnement trophique (biotique), qui peut être défini par les interactions entre espèces (notamment les arbres de la ripisylve source de nourriture via fruits, graines, feuilles, ...) et notamment les relations proie-prédateur au cours des stades successifs du développement. L'ensemble de ces contraintes caractérise l'habitat et doit être considéré dans une dynamique spatiale et temporelle : de la synchronisation entre les besoins d'une écophase et les caractéristiques du milieu dépend la survie d'une cohorte ou de la population.

En ce qui concerne les poissons, les proportions relatives des concentrations en ions majeurs semblent sans influence particulière sur les peuplements. Si une salinité élevée peut être mise à l'évidence une contrainte limitant la diversité des poissons dans un milieu, cela n'est pas le cas pour les très faibles salinités pour lesquelles un 
éventuel effet sélectif n'est pas encore démontré (Lemoalle, 2006).

La fluctuation des facteurs abiotiques et biotiques est souvent responsable de certains changements constatés au sein des communautés en milieux aquatiques continentaux. Ces changements sont liés à la fluctuation des caractéristiques physicochimiques du milieu, notamment le substrat, la disponibilité en nutriments (Hart et al., 2003), la minéralisation (Piscart et al., 2005), la charge en matière organique (Negishi et Richardson, 2003), le taux en oxygène dissous (Brodersen et al., 2004 ; Tarr et al., 2005) et la diversité des microhabitats accessibles (Jahan et al., 2001).

Il apparaît donc ici clairement que les paramètres comme le régime hydrologique, la vitesse du courant, la sinuosité, la végétation, le type de substrats, la nature des berges ainsi que la densité des bois morts sont des paramètres qui, à des degrés divers, ont déterminé la distribution spatio-temporelle des peuplements piscicoles dans les différentes stations d'échantillonnage. Toutefois, dans certaines stations telles que Yoko 2 et 3, proches de la route, on n'a pas capturé de Mormyridae étant donné que ces stations subissent fréquemment des pressions anthropiques. Les Mormyridae sont parmi les premiers poissons à réagir lors d'altérations $\mathrm{du}$ milieu et sont, par conséquent, pourraient être considérés comme de bonsbio indicateurs permettant de caractériser l'état présent d'un habitat-clé (Dale et Beyeler, 2001; Niemi et McDonald, 2004), ainsi que l'évolution de l'état de l'écosystème dans le temps (Lévêque et Mounolou, 2008 ; Niamien-Ebrottié et al., 2008).

\section{Conclusion}

La répartition des espèces par station montre qu'il existe des liens de similarité et /ou un ensemble complexe d'interrelations entre les différentes variables de plusieurs ordres qui décrivent l'environnement des stations et les espèces présentes. Toutefois, la diversité des milieux et la variété des biotopes ne peuvent être définies de façon simple que si l'on privilégie une approche comparative. Celle-ci peut porter sur les caractéristiques hydro-morphologiques des stations des cours d'eau et leur relation avec le métabolisme des espèces présentes et leurs exigences alimentaires et reproductives. Ceci explique une richesse taxonomique la plus élevée aux stations ayant une diversité d'habitats dont la nature de fond du substrat est garnie de sables, galets, graviers, branchages, feuillages, vases et limons (Yoko 4 et Biaro 3 avec respectivement 6 et 5 espèces et indice d'équitabilité élevé. Cela est dû au fait que ces deux stations se trouvent en aval des principaux cours d'eau et situées en pleine réserve où les conditions écologiques sont stables. Une étude plus fine des microhabitats, accompagné de la caractérisation de la valence écologique des espèces pourrait apporter plus de lumière sur l'abondance relative des espèces de Mormyridae dans ces rivières.

\section{CONFLIT D'INTERETS}

Les auteurs déclarent qu'il n'y a aucun conflit d'intérêt.

\section{CONTRIBUTIONS DES AUTEURS}

RMB est l'investigateur principal. JNN a réalisé la récolte des données, AKB, JUA-P et J-CM ont supervisé les travaux.

\section{REMERCIEMENTS}

Nous remercions tous ceux qui ont contribué d'une manière ou d'une autre à la réalisation de ce travail. Ainsi, nous pensons à l'Université de Namur/Belgique et à la Faculté des Sciences de l'Université de Kisangani pour l'encadrement et la supervision sans oublier les amis avec qui nous avons souffert pendant la récolte des données.

\section{REFERENCES}

Abell R, Thieme ML, Revenga C, Bryer M, Kottelat M, Bogutskaya N, Coad B, Mandrak N, Balderas CS, Bussing W, Melaniel JS, Skelton P, Gerald R, Allen, Unmack P, Naseka A, Rebecca Ng, 
Sindorf N, Robertson J, Armijo E, Jonathan V, Higgins, Thomas J, Heibel, Wikramanayake E, Olson D, López IH, Roberto E, Reis, John G, Lundberg, Mark H, Sabajpérez and Petry P. 2008. Freshwater Ecoregions of the world: A New Map of Biogeographic Units for Freshwater Biodiversity Conservation. BioScience, 58(5): 403 - 414. DOI : http://www.biosciencemag.org

Aboua RDB, N'zi konan G, Kouamelan PE, Berte S, Bamba M. 2010. Organisation spatiale du peuplement de poissons dans le Bandama. Int. J. Biol. Chem. Sci., 4(5): 1480-1493. DOI : http://ajol.info/index.php/ijbcs

Agence de l'eau Rhin. Meuse. 2000. Notice d'utilisation de la fiche « description du milieu physique $»$, Rhin. , Berlin.

Alhou B. 2007. Impact des rejets de la ville de Niamey sur la qualité des eaux du fleuve Niger. $\mathrm{PhD}$ thèse en Sciences, Presses universitaires de Namur. FUNDP, Namur, p. 230.

Angelier E. 2000. Ecologie des Eaux Courantes. Editions Technique \& Documentation : Paris.

Ashley B, Robin A. 2008. Ecoregion 539: Upper Congo Rapids in Freshwater ecoregion of the world. Conservation Science Program, WWF-US: Washington, DC, USA., 2 p.

Brodersen KP, Pedersen O, Lindegaard C, Hamburger K. 2004. Chironomids (Diptera) and oxy-regulatory capacity: An experimental approach to paleolimnological interpretation. Limnology and Oceanography, 49(5): 1549-1559. DOI: http:// m.aslo.info/lo/toc/vol_49/issue_5/1549.p df

Bütler R. 2000. Analyse de la distribution spatiale d'objets dans un paysage Fiche d'enseignement, Labo. De gestion des écosystèmes (GECOS) : Lausanne.

Da Costa KS, Gourène G, Tito De Morais L, Thys Van Den Audenaerde DFE. 2000. Caractérisation des peuplements ichtyologiques de deux fleuves côtiers
Ouest-Africains soumis à des aménagements hydroagricoles et hydroélectriques. Vie et Milieu, 50 : 6577 DOI :https://www.kmae-journal.org; https://doi.org/10.1051/kmae/2012002; https://scholar.google.com/citations?user $=$ BhzUOocAAAAJ\&hl $=\mathrm{fr}$

Dajoz R. 1996. Précis d'Écologie (6 ème édn). Dunod : Paris.

Dale VH, Beyeler SC. 2001. Challenges in the development and use of ecological indicators. Ecol. Indic., 1: 3-10. DOI: http://www.napawatersheds.org

Hart RC, Campbell LM, Hecky RE. 2003. Stable isotope analyses and demographic responses counter prospects of planktivory by Caridina (Decapoda: Atyidae) in Lake Victoria. Oecologica, 136: $270-278$. DOI $10.1007 / \mathrm{s} 00442-003-$ 1261-0; http://ap.smu.ca/ lcampbel/ HartetalOecol2003.pdf

Hugueny B, Lévêque C. 2006. Richesse en espèces des peuplements de poissons. In Les Poissons des Eaux Continentales Africaines: Diversité, Écologie, Utilisation par l'Homme, Lévêque, Christian, Paugy D (eds). Edition IRD : Paris ; 271-284.

Jahan S, Akter S, Sarker M, Rahman R, Pramanik. 2001. Growth ecology of Pila globosa (Swainson) (Gastropoda: Pilidae) insimulated habitat. Pakistan Journal of Biological Sciences, 4(5): 581-584. DOI: http://www.pjbs.org m

Jongman RH, terBraak CJF, van Tongeren OFR. 1995. Data Analysis Community and Landscape Ecology. Cambridge University Press: Cambridge.

Kankonda B. 2007 : Ecologie des Décapodes des ruisseaux de forêt de basse altitude de Kisangani (R. D. Congo): Composition, distribution spatiotemporelle, abondance, biomasse et production. Cas du ruisseau Masangamabe de la Reserve Forestière de Masako. PhD thèse in éd. Fac. Sc. Dpt. Hydrobiol., Unikis, Kisangani, p. 202. 
Kerckhove O. 2012. Espèces ou association d'espèces de poissons en tant que bio indicateur de l'état de santé des récifs coralliens. Mém. de maîtrise inédit, Fac. Sc. Université de Sherbrooke : Québec.

Kouadio NF, Kouamélan EP, N'Douba V, Koné T, Snoeks J, Ollevier F. 2006. Update of fish biodiversity and impact of human activities on the community structure, Mé River (Ivory Coast). Int. J. Biol. Chem. Sci., 6: 805-814. DOI: https://www.ajol.info/index.php/ijbcs/art icle/download/65547/53234

Kouamé KA, Yao SS, Gooré Bi G, Kouamélan EP, N'Douba V, Kouassi NJ. 2008. Influential environmental gradients and patterns of fish assemblages in a West African basin. Hydrobiologia, 603: 159-169. DOI: http://dx.doi.org/10.1051/kmae/2012002

Kouamélan EP, Teugels GG, N'Douba V, Gooré BIG, Koné T. 2003. Fish diversity and its relationship with environment variables in West African basin. Hydrobiologia, 505: 139-146. DOI: 10.1023/B:HYDR.0000007302.74296.84; https://www.ncbi.nlm.nih.gov/pmc/articl es/PMC3506418/

Kramer B, in Binder MD, Hirokawa N, Windorst U. 2009. Encyclopedia of Neuroscience. Springer: Berlin Heidelberg.

Lalèyè $\mathrm{P}$, Chikou A, Philippart J-C, Teugels G, Vandewalle P. 2004. Étude de la diversité ichtyologiquedu bassin du fleuve Ouémé au Bénin (Afrique de l'Ouest). Cybium, 28(4): 329-339. DOI: http://sfi.mnhn.fr/cybium/numeros/2004/ 284/05.

Kumba S, Nshimba H, Ndjele L, De Cannière C, Visser M, Bogaert J. 2013. Structure spatiale des trois espèces les plus abondantes dans la Réserve Forestière de la Yoko, Ubundu, République Démocratique du Congo. Tropicultura, 31(1): $53-61 . \quad$ DOI : http://www.tropicultura.org/text/v31n1.p df
Legendre P, Legendre L. 1998. Numerical Ecology ( $2^{\text {nd }}$ edn). Elsevier Science B.V.: Amsterdam.

Lejoly J, Ndjele BM, Geerinck D. 2010. Catalogue-Flore des Plantes Vasculaires des Districts de Kisangani et de la Tshopo (RD Congo), ( $4^{\text {ème }}$ edn). Taxonomania: Bruxelles.

Lemoalle J. 2006. La Diversité des milieux aquatiques. In Les Poissons des Eaux Continentales Africaines: Diversité, Écologie, Utilisation par l'Homme, Lévêque, Christian, Paugy D (eds). Edition IRD : Paris ; 11-30.

Lévèque C, Paugy D. 2006. Peuplements des cours d'eau et des biotopes. In Les Poissons des Eaux Continentales Africaines: Diversité, Écologie, Utilisation par l'Homme, Lévêque, Christian, Paugy D (eds). Edition IRD : Paris ; 317-328.

Lévêque C, Mounolou J-C. 2008. Biodiversité : Dynamique Biologique et Conservation ( $2^{\text {ème }}$ édn). Dunod: Paris.

Mbega JD. 2004. Biodiversité des poissons du bassin inférieur de l'Ogoué (Gabon). Volume 1. PhD thèse de doctorat, FUNDP/Namur, Namur, p. 324.

Mbega JD. 2004. Biodiversité des Poissons du Bassin Inférieur de l'Ogooué (Gabon). (VolI et II). Presses Universitaires de Namur: Namur.

Mbimbi M. 2006. Contribution à l'étude de la biologie et de l'écologie de Marcusenius greshoffi (Schilthuis, 1891). Mémoire de DEA. UNIKIN : Kinshasa.

Niamien-Ebrottié EJ, Konan KF, Gnagne T, Ouattara A, Ouattara M, Gourène G. 2008. Etude diagnostique de l'état de pollution du système fluvio-lagunaire Aby-Bia-Tanoé (Sud-Est, Côte d'Ivoire). Sud Sciences et Technologies, 16(1): 513. DOI : http://documents.irevues.inist. fr/bitstream/handle/2042/30379/sst16_2.

Niemi GT, McDonald ME. 2004. Application of ecological indicators. Annu. Rev. Ecol. Evol. Syst., 35: 89-111. DOI: 10.1146/annurev.ecolsys.35.112202.130 132 ; 
Paugy D, Lévêque C, Teugels GG. 2003. Poissons d'Eaux Douces et Saumâtres de l'Afrique de l'Ouest (Tome I et II). IRD, MNHN et MRAC: Paris.

Piélou C. 1969. An Introduction to Mathematical Ecology (Vol VIII). John Wiley \& Sons: New York.

Pigneur LM. 2005. Taxonomie du genre Marcusenius (Mormyridae) au pool Malebo (Kinshasa, R.D. Congo) et contribution à l'étude de la biologie et l'écologie de Marcusenius macrolepidotus angolensis. Mémoire inédit, FUNP/Namur.

Piscart C. Moreteau J.-C, Beisel J.-N. 2005. Biodiversity and structure of macro invertebrate communities along a small permanent salinity gradient (Meurthe River, France). Hydrobiologia, 551(1): 227-236. DOI10.1007/s10750-005-44630; https://www.researchgate.net

Poll M, Gosse JP. 1995. Généra des Poissons d'Eau Douce de l'Afrique (eds). Académie Royale de Belgique : Bruxelles.

Sullivan JP, Hopkins CD. 2001. Quand les poissons apportent leurs premières pierres et leurs premiers signaux électriques, Corneluniversity, USA; 1 - 4.

Sullivan JP, Lavoué S, Friel JP. 2004. A la découverte des poissons du parc national
d'Odzala. Canopée Bulletin sur l'Environnement en Afrique Centrale, 17-21. http://pages.nbb.cornell.edu/ neurobio/hopkins/Reprints/2605_Poisson sOdzalaCon

Tarr TL, Baber MJ, Babbitt. 2005. Macroinvertebrate community structure across a wetland hydroperiod gradient in southern New Hampshire, USA. Wetlands Ecology and Management, 13(3): 321-334. DOI: 10. 1007/s11273004-7525-6; https://insights.ovid.com/ wetlands-ecology-management/ wetem/2005/06/000/macr.

Verniers G, Duchâtel J, Hardy MA. FUNDPGIREA. 2009. Evaluation de la Qualité Physique des Cours d'Eau : Application du «Qualphy » au Bassin de la Gette. GIREA : Namur.

Verniers G. 2005. La Rivière Milieu Vivant. GIREA: Namur.

Yao SS, Kouamélan EP, Koné T, N'Douba V, Gooré Bi G, Ollevier F, Thys Van Den Audenaerde DFE. 2005. Fish communities along environment gradients within the Comoé River basin, Côte d'Ivoire. African Journal of AquaticScience, 30: 195-204. DOI: https://doi.org/10.1051/kmae/2012002. 